\title{
Computational strategies for national integration of phenotypic, genomic, and pedigree data in a single-step best linear unbiased prediction
}

\author{
A. Legarra* ${ }^{1}$ and V. Ducrocq $\dagger$ \\ *INRA, UR 631 SAGA, F-31326 Castanet Tolosan, France \\ †INRA, UMR1313 GABI, F-78352 Jouy en Josas, France
}

28,800 individuals and 1,800 genotyped individuals, all methods converged quickly to the same solutions. Using existing efficient methods with limited memory requirements to compute the products $\mathbf{G t}$ and $\mathbf{A}_{22} \mathbf{t}$ for any $\mathbf{t}$ (where $\mathbf{G}$ and $\mathbf{A}_{22}$ are genomic and pedigree relationships for genotyped animals, and $\mathbf{t}$ is a vector), all strategies can be converted to iteration on data procedures for which the total number of operations is linear in the number of animals + number of genotyped animals $\times$ number of markers.

Key words: genetic evaluation, genomic selection, mixed model

\section{INTRODUCTION}

Genetic evaluations in dairy cattle nowadays include massive SNP marker data. This will be referred to as genomic evaluation hereinafter. Methods include adaptations of BLUP evaluations, sophisticated regressions based mainly on Bayesian models, and nonparametric techniques. Results have generally shown a superiority of genomic evaluations over pedigree-based ones.

In pedigree-based models using BLUP, large data sets are used. Solution of mixed-model equations (MME) usually takes $\mathrm{O}(n)$ computing time; that is, central processing unit (CPU) time roughly proportional to $n$, the number individuals in the pedigree, using iteration on data techniques that reduce storage needs to $\mathrm{O}(n)$ elements (Schaeffer and Kennedy, 1986; Misztal and Gianola, 1987; Strandén and Lidauer, 1999). Genomic evaluations that use mixed models, such as Bayesian regressions, Lasso, or Elastic Net, can be efficiently computed by several strategies, with an $\mathrm{O}(p m)$ cost, where $p$ is the number of genotyped individuals and $m$ is the number of SNP effects (Legarra and Misztal, 2008; VanRaden, 2008; Friedman et al., 2010; Shepherd et al., 2010). Iteration on data techniques can reduce storage to $\mathrm{O}(m)$ variables.

However, all of these strategies work for models where the own phenotype of the genotyped individual is being analyzed. In dairy cattle, daughter yield deviations (DYD; VanRaden and Wiggans, 1991) are used.

Received September 27, 2011.

Accepted April 3, 2012.

${ }^{1}$ Corresponding author: andres.legarra@toulouse.inra.fr 
Unfortunately, bias in computation of DYD resulting from genomic preselection of the best animals may become a problem (Mäntysaari and Strandén, 2010; Patry and Ducrocq, 2011a,b). Other problems of DYD are their variable accuracy, difficult definition, or computation for some nonlinear traits (e.g., longevity) or species (e.g., sheep or swine).

A solution to this problem is the one- or single-step method, suggested simultaneously by Misztal et al. (2009), Legarra et al. (2009), Aguilar et al., (2010), and Christensen and Lund (2010). We propose here to call this method single-step genomic BLUP (SSGBLUP). The method uses the property that, at a given locus, the covariance of the gene frequency $(g=\{0,1 / 2,1\})$ for the $i$ th allele of 2 individuals $j$ and $k$ is described by the expression $\operatorname{Cov}\left(g_{i j}, g_{i k}\right)=\theta_{j k} p_{i}\left(1-p_{i}\right)$ (Cockerham, 1969), where $\theta_{j k}$ is the coancestry of the 2 individuals. Thus, the (unobserved) genotype of an individual can be modeled using the numerator relationship matrix (Gengler et al., 2007; Christensen and Lund, 2010). This will equally be true for any linear function of genotypes (such as additive breeding values, which are a linear function of genotypes at causal loci). Therefore, the expression can be applied to breeding values, markers, or haplotypes, and warrants generality of the derivations of SSGBLUP to models based on markers, haplotypes, or even nonparametric genomic covariances.

Consider the model

$$
\mathbf{y}=\mathbf{X b}+\mathbf{W u}+\mathbf{e},
$$

where $\mathbf{y}$ is a vector of phenotypes at a trait; $\mathbf{b}, \mathbf{u}$, and e are fixed effects, random effects, and residuals, respectively; and $\mathbf{X}$ and $\mathbf{W}$ are incidence matrices.. For clarity and without loss of generality, we will consider here that $\mathbf{R}=\mathbf{I} \sigma_{e}^{2}$, where $\mathbf{R}$ is the covariance matrix of $\mathbf{e}, \mathbf{I}$ is an identity matrix, and $\sigma_{e}^{2}$ is the residual variance.

The MME for single-trait SSGBLUP are

$$
\begin{gathered}
{\left[\begin{array}{cc}
\mathbf{X}^{\prime} \mathbf{X} & \mathbf{X}^{\prime} \mathbf{W} \\
\mathbf{W}^{\prime} \mathbf{X} & \mathbf{W}^{\prime} \mathbf{W}+\alpha_{u} \mathbf{H}^{-1}
\end{array}\right]\left[\begin{array}{l}
\hat{\mathbf{b}} \\
\hat{\mathbf{u}}
\end{array}\right]=} \\
{\left[\begin{array}{c}
\mathbf{X}^{\prime} \mathbf{y} \\
\mathbf{W}^{\prime} \mathbf{y}
\end{array}\right]}
\end{gathered}
$$

with terms as previously defined, and

$$
\mathbf{H}^{-1}=\left[\begin{array}{cc}
\mathbf{A}^{11} & \mathbf{A}^{12} \\
\mathbf{A}^{21} & \mathbf{A}^{22}+\mathbf{G}^{-1}-\mathbf{A}_{22}^{-1}
\end{array}\right],
$$

where $\mathbf{A}$ and $\mathbf{G}$ are, respectively, pedigree-based (numerator) and genomic relationship matrices, partitioned in genotyped and ungenotyped individuals, $\sigma_{u}^{2}=\operatorname{Var}(u)$ is the polygenic genetic variance and $\alpha_{u}=\sigma_{e}^{2} / \sigma_{u}^{2}$. The model is easily extended to a multiple trait or a model including maternal or random regression genetic effects by including a more general structure in $\mathbf{R}$, substituting $\sigma_{u}^{2}$ by a covariance matrix, and using Kronecker products. The $\mathbf{G}$ matrix is usually formed as in VanRaden (2008); that is, $\mathbf{G}=\mathbf{Z D Z}^{\prime}$, where $\mathbf{Z}$ is a centered incidence matrix of SNP, $\mathbf{D}_{i, i}=$ $m / 2 p_{i} q_{i}$ or $\mathbf{D}_{i, i}=1 / 2 \Sigma p_{i} q_{i}$, and $p_{i}$ is the allelic frequency of the $i$ th $(i=1 \ldots m)$ locus. An improved $\mathbf{G}$ may require an appropriate scaling of $\mathbf{G}$ so that $\mathbf{G}$ and $\mathbf{A}_{22}$ are proportional to the same variance component $\sigma_{u}^{2}$ (VanRaden, 2008; Vitezica et al., 2011). Computation strategies for SSGBLUP are described in Aguilar et al. $(2010,2011)$. The cost of inversions of $\mathbf{G}$ and $\mathbf{A}_{22}$ is a cubic function $\mathrm{O}\left(p^{3}\right)$ of the number of genotyped animals $(p)$.

In our opinion, 3 major barriers for widespread use of SSGBLUP are (1) this (cubic) computing cost; (2) the need to modify the existing software, which covers the wide variety of evaluation models currently used (including heterogeneity of variances, multiple-trait models, threshold models, test-day models, and survival models, among others); and (3) its linear form, which precludes the use of nonlinear estimators including genomic information. We address here 2 of these 3 problems. We show how to write 2 sets of equations equivalent to Equation 2, computable in $\mathrm{O}(n)+\mathrm{O}(p m)$, without explicit inversion and we present an alternative derivation of Equation 2, leading to an iterative solution procedure that allows the use of already written BLUP software, which cannot otherwise incorporate genomic information. Both allow iteration on data techniques. As for the third problem, a partial solution is to precompute an improved $\mathbf{G}$ matrix (Zhang et al., 2010; Legarra et al., 2011). We outline a more general procedure to include Bayesian estimators, based on the iterative approach.

\section{MATERIALS AND METHODS}

We propose to solve Equation 2 by iteratively considering 2 systems: the first one as close as possible to the regular MME, apart from a correction of the right-hand side, and the second one isolating the block corresponding to genotyped animals. In other words, an iterative solution of Equation 2 is obtained, alternating between a regular genetic evaluation and a regular genomic 
evaluation, whatever the model and the methodology chosen for either one. Such strategy should lead to only minimal modifications of the existing BLUP software used for national evaluations and should be compatible with most genomic evaluation methods.

Two different derivations of these 2 systems will be presented. Although their starting point is quite different, they lead to equivalent results.

\section{Extended SSGBLUP MME Introducing Additional Unknowns}

The first technique is the derivation of an equivalent model by including additional unknowns. This form of data augmentation can be seen as the opposite of the well-known absorption technique, and aims at simplifying computations. For instance, Henderson (1977) showed that including all animals in a pedigree in the set of unknowns allowed the use of his simple rules for inversion of A (Henderson, 1976). Similarly, including a permanent environment effect in a simple repeatability model leads to an easier solution than explicitly using the residual covariance structure between repeated records of a same animal.

Distinguishing between phenotypes of nongenotyped and genotyped animals, we can write Equation 1 as

$$
\left[\begin{array}{l}
\mathbf{y}_{1} \\
\mathbf{y}_{2}
\end{array}\right]=\left[\begin{array}{l}
\mathbf{X}_{1} \\
\mathbf{X}_{2}
\end{array}\right] \mathbf{b}+\left[\begin{array}{cc}
\mathbf{W}_{1} & \mathbf{0} \\
\mathbf{0} & \mathbf{W}_{2}
\end{array}\right]\left[\begin{array}{l}
\mathbf{u}_{1} \\
\mathbf{u}_{2}
\end{array}\right]+\left[\begin{array}{l}
\mathbf{e}_{1} \\
\mathbf{e}_{2}
\end{array}\right] .
$$

Regular SSGBLUP. Consider then an extended form of system [2]:

$$
\begin{gathered}
{\left[\begin{array}{ccc}
\mathbf{X}^{\prime} \mathbf{X} & \mathbf{X}_{1}^{\prime} \mathbf{W}_{1} & \mathbf{X}_{2}^{\prime} \mathbf{W}_{2} \\
\mathbf{W}_{1}^{\prime} \mathbf{X}_{1} & \mathbf{W}_{1}^{\prime} \mathbf{W}_{1}+\alpha_{u} \mathbf{A}^{11} & \alpha_{u} \mathbf{A}^{12} \\
\mathbf{W}_{2}^{\prime} \mathbf{X}_{2} & \alpha_{u} \mathbf{A}^{12} & \mathbf{W}_{2}^{\prime} \mathbf{W}_{2}+\alpha_{u} \mathbf{A}^{22}+\alpha_{u}\left(\mathbf{G}^{-1}-\mathbf{A}_{22}^{-1}\right)
\end{array}\right]} \\
{\left[\begin{array}{c}
\hat{\mathbf{b}} \\
\hat{\mathbf{u}}_{1} \\
\hat{\mathbf{u}}_{2}
\end{array}\right]=\left[\begin{array}{c}
\mathbf{X}^{\prime} \mathbf{y} \\
\mathbf{W}_{1}^{\prime} \mathbf{y}_{1} \\
\mathbf{W}_{2}^{\prime} \mathbf{y}_{2}
\end{array}\right],}
\end{gathered}
$$

where, again, subscripts 1 and 2 refer to nongenotyped and genotyped animals, respectively, and $\mathbf{X}^{\prime}=$ $\left(\mathbf{X}_{1}{ }^{\prime} \mathbf{X}_{2}{ }^{\prime}\right)$. Complex models (e.g., random regression or maternal models) would include terms such as $\mathbf{W}_{1}{ }^{\prime} \mathbf{W}_{2}$, but this will not be used here (although our derivations are completely general). In Equation 3, u was split in ungenotyped $\left(\mathbf{u}_{1}\right)$ and genotyped $\left(\mathbf{u}_{2}\right)$ animals.
Unsymmetric Extended SSGBLUP. An equivalent system of equations is

$$
\begin{gathered}
{\left[\begin{array}{ccccc}
\mathbf{X}^{\prime} \mathbf{X} & \mathbf{X}_{1}^{\prime} \mathbf{W}_{1} & \mathbf{X}_{2}^{\prime} \mathbf{W}_{2} & \mathbf{0} & \mathbf{0} \\
\mathbf{W}_{1}^{\prime} \mathbf{X}_{1} & \mathbf{W}_{1}^{\prime} \mathbf{W}_{1}+\alpha_{u} \mathbf{A}^{11} & \alpha_{u} \mathbf{A}^{12} & \mathbf{0} & \mathbf{0} \\
\mathbf{W}_{1}^{\prime} \mathbf{X}_{2} & \alpha_{u} \mathbf{A}^{12} & \mathbf{W}_{2}^{\prime} \mathbf{W}_{2}+\alpha_{u} \mathbf{A}^{22} & \alpha_{u} \mathbf{I} & -\alpha_{u} \mathbf{I} \\
\mathbf{0} & \mathbf{0} & \alpha_{u} \mathbf{I} & \alpha_{u} \mathbf{A}_{22} & \mathbf{0} \\
\mathbf{0} & \mathbf{0} & \alpha_{u} \mathbf{I} & \mathbf{0} & \alpha_{u} \mathbf{G}
\end{array}\right]} \\
{\left[\begin{array}{c}
\hat{\mathbf{b}} \\
\hat{\mathbf{u}} \\
\hat{\mathbf{u}}_{2} \\
-\hat{\mathbf{\varphi}} \\
-\hat{\boldsymbol{\gamma}}
\end{array}\right]}
\end{gathered}
$$

where $\varphi$ and $\gamma$ are new vectors of unknowns, with the same size as $\mathbf{u}_{2}$. Vector $\gamma$ corresponds to vector $\mathbf{s}$ in Strandén and Garrick (2009). The lack of symmetry of these equations will be used later to describe the convergence of iterative methods to the solution. Absorption of the last 2 equations into the first 3 equations leads to system [3]. Equation 4 can be solved, for instance, by successive underrelaxation (SUR; Broyden, 1964; which is a special case of successive overrelaxation (SOR) with relaxation factors lower than 1; we will return to this point later) or the biconjugate gradient stabilized method (BiCGstab; van der Vorst, 2003; Misztal et al., 2009). If one changes the sign of the bottom row of Equation 4, this creates a symmetric system of equations. In that case, a negative sign is associated with $\mathbf{G}$, which is unusual; this does not prevent $\gamma$ from being a variable with real domain. It is, in fact, a linear transformation of $\mathbf{u}$; actually, $\boldsymbol{\gamma}=\mathbf{G}^{-1} \mathbf{u}_{2}, \quad \boldsymbol{\varphi}=\mathbf{A}_{22}^{-1} \mathbf{u}_{2}, \quad$ with variances $\operatorname{Var}(\boldsymbol{\gamma})=\mathbf{G}^{-1} \sigma_{u}^{2}, \operatorname{Var}(\boldsymbol{\varphi})=\mathbf{A}_{22}^{-1} \sigma_{u}^{2}$.

\section{Extended SSGBLUP MME Introducing Additional Unknowns and Marker Effects}

For models where $\mathbf{G}=\mathbf{Z D Z}^{\prime}$, another set of equations explicit on marker solutions $\hat{\mathbf{g}}$ is as follows: 


$$
\begin{gathered}
{\left[\begin{array}{ccccc}
\mathbf{X}^{\prime} \mathbf{X} & \mathbf{X}_{1}^{\prime} \mathbf{W}_{1} & \mathbf{X}_{2}^{\prime} \mathbf{W}_{2} & \mathbf{0} & \mathbf{0} \\
\mathbf{W}_{1}^{\prime} \mathbf{X}_{1} & \mathbf{W}_{1}^{\prime} \mathbf{W}_{1}+\alpha_{u} \mathbf{A}^{11} & \alpha_{u} \mathbf{A}^{12} & \mathbf{0} & \mathbf{0} \\
\mathbf{W}_{1}^{\prime} \mathbf{X}_{2} & \alpha_{u} \mathbf{A}^{12} & \mathbf{W}_{2}^{\prime} \mathbf{W}_{2}+\alpha_{u} \mathbf{A}^{22} & \alpha_{u} \mathbf{I} & -\alpha_{u} \mathbf{G}^{-1} \mathbf{Z} \\
\mathbf{0} & \mathbf{0} & \alpha_{u} \mathbf{I} & \alpha_{u} \mathbf{A}_{22} & \mathbf{0} \\
\mathbf{0} & \mathbf{0} & -\alpha_{u} \mathbf{Z}^{\prime} \mathbf{G}^{-1} & \mathbf{0} & -\alpha_{u} \mathbf{D}^{-1}
\end{array}\right]} \\
{\left[\begin{array}{c}
\hat{\mathbf{b}} \\
\hat{\mathbf{u}}_{1} \\
\hat{\mathbf{u}}_{2} \\
-\hat{\mathbf{\varphi}} \\
-\hat{\mathbf{g}}
\end{array}\right]=\left[\begin{array}{c}
\mathbf{X}^{\prime} \mathbf{y} \\
\mathbf{W}_{1}^{\prime} \mathbf{y}_{1} \\
\mathbf{W}_{2}^{\prime} \mathbf{y}_{2} \\
\mathbf{0} \\
\mathbf{0}
\end{array}\right] .}
\end{gathered}
$$

These equations show that $\hat{\mathbf{g}}=\mathbf{D Z}^{\prime} \mathbf{G}^{-1} \hat{\mathbf{u}}_{2}$, which is the best linear predictor of marker effects $\mathbf{g}$ given $\mathbf{u}_{2}$ (Henderson, 1973; Strandén and Garrick, 2009). Similar systems of equations are being used by some scientists [P. M. VanRaden, US Department of Agriculture, Bethesda, MD, personal communication; D. L. Johnson, Livestock Improvement Corp. (LIC), Hamilton, New Zealand, personal communication; N. Gengler, Université de Liège (ULg), Gembloux, Belgium, personal communication]. A reduced, but possibly more cumbersome, system of equations including $\mathbf{u}_{1}$ and $\mathbf{g}$ (but not $\mathbf{u}_{2}$ ) is as follows:

$$
\begin{aligned}
& {\left[\begin{array}{cccc}
\mathbf{X}^{\prime} \mathbf{X} & \mathbf{X}_{1}^{\prime} \mathbf{W}_{1} & \mathbf{X}_{2}^{\prime} \mathbf{W}_{2} \mathbf{Z} & \mathbf{0} \\
\mathbf{W}_{1}^{\prime} \mathbf{X}_{1} & \mathbf{W}_{1}^{\prime} \mathbf{W}_{1}+\alpha_{u} \mathbf{A}^{11} & \alpha_{u} \mathbf{A}^{12} \mathbf{Z} & \mathbf{0} \\
\mathbf{Z}^{\prime} \mathbf{W}_{2}^{\prime} \mathbf{X}_{2} & \alpha_{u} \mathbf{Z}^{\prime} \mathbf{A}^{12} & \mathbf{Z}^{\prime} \mathbf{W}_{2}^{\prime} \mathbf{W}_{2} \mathbf{Z}+\alpha_{u} \mathbf{Z}^{\prime} \mathbf{A}^{22} \mathbf{Z}+\mathbf{D}^{-1} \sigma_{e}^{2} & \alpha_{u} \mathbf{Z}^{\prime} \\
\mathbf{0} & \mathbf{0} & \alpha_{u} \mathbf{Z} & \alpha_{u} \mathbf{A}_{22}
\end{array}\right.} \\
& {\left[\begin{array}{c}
\hat{\mathbf{b}} \\
\hat{\mathbf{u}}_{1} \\
\hat{\mathbf{g}} \\
-\hat{\mathbf{\varphi}}
\end{array}\right]=\left[\begin{array}{c}
\mathbf{X}^{\prime} \mathbf{y} \\
\mathbf{W}_{1}^{\prime} \mathbf{y}_{1} \\
\mathbf{Z}^{\prime} \mathbf{W}_{2}^{\prime} \mathbf{y}_{2} \\
\mathbf{0}
\end{array}\right]}
\end{aligned}
$$

In contrast to the previous one, the left-hand side of these equations is positive definite and symmetric. Its derivation is presented in Appendix A.

\section{Extended SSGBLUP MME Derived from an Equivalent Model}

In model 1 , one can decompose $\mathbf{u}$ into a strictly polygenic part $\mathbf{u}^{*}$ and a deviation due to the genomic information that we will call $\mathbf{d}=\mathbf{u}-\mathbf{u}^{*}$ :

$$
\mathbf{y}=\mathbf{X b}+\mathbf{W}\left(\mathbf{u}^{*}+\mathbf{d}\right)+\mathbf{e} .
$$

Let us assume (or impose) that $\operatorname{Var}\left(\begin{array}{c}\mathbf{u}^{*} \\ \mathbf{d}\end{array}\right)=\left(\begin{array}{cc}\mathbf{A} & \mathbf{0} \\ \mathbf{0} & \mathbf{G}-\mathbf{A}\end{array}\right) \sigma_{u}^{2}$. Although $\mathbf{G}$ is guaranteed to be semipositive definite and $\mathbf{A}$ is positive definite, $\mathbf{G}-\mathbf{A}$ (although full rank) has no guarantees whatsoever; in fact, more often than not, the eigenvalues of $\mathbf{G}-\mathbf{A}$ span negative and positive values. Thompson (1979) and Thompson and Meyer (1990) suggested including imaginary effects to deal with negative variances. These equivalent models were found to considerably reduce computing time for estimation of genetic parameters with a multivariate reduced animal model (Besbes et al., 1992). Developing this idea, in Appendix $\mathrm{C}$ (which we suggest reading after Appendix B) we show that mixed model equations are identical whether $\mathbf{G}-\mathbf{A}$ is positive definite or not, as far as it is invertible.

Distinguishing between phenotypes of nongenotyped and genotyped animals, we can write Equation 1 as

$$
\left[\begin{array}{l}
\mathbf{y}_{1} \\
\mathbf{y}_{2}
\end{array}\right]=\left[\begin{array}{l}
\mathbf{X}_{1} \\
\mathbf{X}_{2}
\end{array}\right] \mathbf{b}+\left[\begin{array}{cc}
\mathbf{W}_{1} & \mathbf{0} \\
\mathbf{0} & \mathbf{W}_{2}
\end{array}\right]\left[\begin{array}{l}
\mathbf{u}_{1}^{*}+\mathbf{d}_{1} \\
\mathbf{u}_{2}^{*}+\mathbf{d}_{2}
\end{array}\right]+\left[\begin{array}{l}
\mathbf{e}_{1} \\
\mathbf{e}_{2}
\end{array}\right] .
$$

For nongenotyped animals, the deviation $\mathbf{d}_{1}$ due to the genomic information is modeled as a linear function of $\mathbf{d}_{2}$, obtained by regressing on genomic contribution $\mathbf{d}_{2}$ from genotyped individuals: $\mathbf{d}_{1}=\mathbf{A}_{12} \mathbf{A}_{22}^{-1} \mathbf{d}_{2}$. Therefore,

$$
\left[\begin{array}{l}
\mathbf{y}_{1} \\
\mathbf{y}_{2}
\end{array}\right]=\left[\begin{array}{l}
\mathbf{X}_{1} \\
\mathbf{X}_{2}
\end{array}\right] \mathbf{b}+\left[\begin{array}{cc}
\mathbf{W}_{1} & \mathbf{0} \\
\mathbf{0} & \mathbf{W}_{2}
\end{array}\right]\left[\begin{array}{l}
\mathbf{u}_{1}^{*} \\
\mathbf{u}_{2}^{*}
\end{array}\right]+\left[\begin{array}{c}
\mathbf{W}_{1} \mathbf{A}_{12} \mathbf{A}_{22}^{-\mathbf{1}} \\
\mathbf{W}_{2}
\end{array}\right] \mathbf{d}_{2}+\left[\begin{array}{l}
\mathbf{e}_{1} \\
\mathbf{e}_{2}
\end{array}\right] .
$$

From above, $\mathbf{u}_{2}^{*}$ and $\mathbf{d}_{2}$ are assumed to be independent $\quad\left[\operatorname{cov}\left(\mathbf{u}_{2}^{*}, \mathbf{d}_{2}\right)=\mathbf{0}\right], \quad \operatorname{var}\left(\mathbf{u}_{2}\right)=\mathbf{G} \sigma_{u}^{2}, \quad$ and $\operatorname{var}\left(\mathbf{d}_{2}\right)=\left(\mathbf{G}-\mathbf{A}_{22}\right) \sigma_{u}^{2}$. $\operatorname{var}\left(\mathbf{d}_{1}\right)=\mathbf{A}_{12} \mathbf{A}_{22}^{-1}\left(\mathbf{G}-\mathbf{A}_{22}\right) \mathbf{A}_{22}^{-1} \mathbf{A}_{21} \sigma_{u}^{2}$. From this model, MME can be derived (details are provided in Appendix B), and are

$$
\begin{gathered}
{\left[\begin{array}{cccc}
\mathbf{X}^{\prime} \mathbf{X} & \mathbf{W}_{1}^{\prime} \mathbf{W}_{1} & \mathbf{X}_{2}^{\prime} \mathbf{W}_{2} & \mathbf{0} \\
\mathbf{W}_{1}^{\prime} \mathbf{X}_{1} & \mathbf{W}_{1}^{\prime} \mathbf{W}_{1}+\alpha_{u} \mathbf{A}^{11} & \alpha_{u} \mathbf{A}^{12} & \mathbf{0} \\
\mathbf{W}_{2}^{\prime} \mathbf{X}_{2} & \alpha_{u} \mathbf{A}^{21} & \mathbf{W}_{2}^{\prime} \mathbf{W}_{2}+\alpha_{u} \mathbf{A}^{22} & -\alpha_{u} \mathbf{A}_{22}^{-1} \\
\mathbf{0} & \mathbf{0} & -\alpha_{u} \mathbf{A}_{22}^{-1} & \alpha_{u}\left\{\mathbf{A}_{22}^{-1}+\left(\mathbf{G}-\mathbf{A}_{22}\right)^{-1}\right\}
\end{array}\right]} \\
{\left[\begin{array}{c}
\hat{\mathbf{b}} \\
\hat{\mathbf{u}}_{1} \\
\hat{\mathbf{u}}_{2} \\
\hat{\mathbf{d}}_{2}
\end{array}\right]=\left[\begin{array}{c}
\mathbf{X}^{\prime} \mathbf{y} \\
\mathbf{W}_{1}^{\prime} \mathbf{y}_{1} \\
\mathbf{W}_{2}^{\prime} \mathbf{y}_{2} \\
\mathbf{0}
\end{array}\right] .}
\end{gathered}
$$

Also, , 
In Appendix B, we show that the absorption of the last equation of system [7] into the first ones leads to system [3]: models [1] and [6] are equivalent.

The last equation of [12] is

$$
\left[\mathbf{A}_{22}^{-1}+\left(\mathbf{G}-\mathbf{A}_{22}\right)^{-1}\right] \hat{\mathbf{d}}_{2}=\mathbf{A}_{22}^{-1} \hat{\mathbf{u}}_{2}
$$

or

$$
\left(\mathbf{G}-\mathbf{A}_{22}\right)^{-1} \hat{\mathbf{d}}_{2}=\mathbf{A}_{22}^{-1}\left(\hat{\mathbf{u}}_{2}-\hat{\mathbf{d}}_{2}\right)=\mathbf{A}_{22}^{-1} \quad \hat{\mathbf{u}}_{2}^{*},
$$

which becomes, after some manipulations,

$$
\hat{\mathbf{d}}_{2}=\hat{\mathbf{u}}_{2}-\mathbf{A}_{22} \mathbf{G}^{-1} \hat{\mathbf{u}}_{2} .
$$

This result implies that $\hat{\mathbf{u}}_{2}^{*}=\mathbf{A}_{22} \mathbf{G}^{-1} \hat{\mathbf{u}}_{2}$. Note also that $\hat{\mathbf{d}}_{2}=\left(\mathbf{I}-\mathbf{A}_{22} \mathbf{G}^{-1}\right) \mathbf{Z} \hat{\mathbf{g}}$ if $\mathbf{u}_{2}=\mathbf{Z g}$.

\section{Iterative Solution of SSGBLUP MME: Iterative SSGBLUP}

Splitting the MME in [4] into 3 parts, one gets the following block iterative solution algorithm:

(1) Solve

$$
\begin{gathered}
{\left[\begin{array}{ccc}
\mathbf{X}^{\prime} \mathbf{X} & \mathbf{X}_{1}^{\prime} \mathbf{W}_{1} & \mathbf{X}_{2}^{\prime} \mathbf{W}_{2} \\
\mathbf{W}_{1}^{\prime} \mathbf{X}_{1} & \mathbf{W}_{1}^{\prime} \mathbf{W}_{1}+\alpha_{u} \mathbf{A}^{11} & \alpha_{u} \mathbf{A}^{12} \\
\mathbf{W}_{2}^{\prime} \mathbf{X}_{2} & \alpha_{u} \mathbf{A}^{21} & \mathbf{W}_{2}^{\prime} \mathbf{W}_{2}+\alpha_{u} \mathbf{A}^{22}
\end{array}\right]\left[\begin{array}{c}
\hat{\mathbf{b}} \\
\hat{\mathbf{u}}_{1} \\
\hat{\mathbf{u}}_{2}
\end{array}\right]=} \\
{\left[\begin{array}{c}
\mathbf{X}^{\prime} \mathbf{y} \\
\mathbf{W}_{1}^{\prime} \mathbf{y}_{1} \\
\mathbf{W}_{2}^{\prime} \mathbf{y}_{2}
\end{array}\right]+\left[\begin{array}{c}
\mathbf{0} \\
\mathbf{0} \\
\alpha_{u} \hat{\boldsymbol{\varphi}}-\alpha_{u} \hat{\gamma}
\end{array}\right]}
\end{gathered}
$$

for $\hat{\mathbf{b}}, \hat{\mathbf{u}}_{1}$, and $\hat{\mathbf{u}}_{2}$.

(2) Solve

$$
\mathbf{A}_{22} \hat{\boldsymbol{\varphi}}=\hat{\mathbf{u}}_{2} \text { and } \mathbf{G} \hat{\boldsymbol{\gamma}}=\hat{\mathbf{u}}_{2} \quad \text { for } \hat{\varphi} \text { and } \hat{\boldsymbol{\gamma}} \text {. }
$$

Equivalently, splitting the MME in [7] leads to the following:

(1) Solve

$$
\begin{gathered}
{\left[\begin{array}{ccc}
\mathbf{X}^{\prime} \mathbf{X} & \mathbf{X}_{1}^{\prime} \mathbf{W}_{1} & \mathbf{X}_{2}^{\prime} \mathbf{W}_{2} \\
\mathbf{W}_{1}^{\prime} \mathbf{X}_{1} & \mathbf{W}_{1}^{\prime} \mathbf{W}_{1}+\alpha_{u} \mathbf{A}^{11} & \alpha_{u} \mathbf{A}^{12} \\
\mathbf{W}_{2}^{\prime} \mathbf{X}_{2} & \alpha_{u} \mathbf{A}^{21} & \mathbf{W}_{2}^{\prime} \mathbf{W}_{2}+\alpha_{u} \mathbf{A}^{22}
\end{array}\right]\left[\begin{array}{c}
\hat{\mathbf{b}} \\
\hat{\mathbf{u}}_{1} \\
\hat{\mathbf{u}}_{2}
\end{array}\right]=} \\
{\left[\begin{array}{c}
\mathbf{X}^{\prime} \mathbf{y} \\
\mathbf{W}_{1}^{\prime} \mathbf{y}_{1} \\
\mathbf{W}_{2}^{\prime} \mathbf{y}_{2}
\end{array}\right]+\left[\begin{array}{c}
\mathbf{0} \\
\mathbf{0} \\
\alpha_{u} \mathbf{A}_{22}^{-1} \hat{\mathbf{d}}_{2}
\end{array}\right] ;}
\end{gathered}
$$

(2) Solve

$$
\left[\mathbf{A}_{22}^{-1}+\left(\mathbf{G}-\mathbf{A}_{22}\right)^{-\mathbf{1}}\right] \hat{\mathbf{d}}_{2}=\mathbf{A}_{22}^{-1} \hat{\mathbf{u}}_{2}
$$

for $\hat{\mathbf{d}}_{2}$, which becomes, after some manipulations comparable to those leading to [7] and using [8]:

$\left(2^{\prime}\right)$ Compute

$$
\mathbf{A}_{22}^{-1} \hat{\mathbf{d}}_{2}=\left(\mathbf{A}_{22}^{-1}-\mathbf{G}^{-1}\right) \hat{\mathbf{u}}_{2}=\hat{\varphi}-\hat{\gamma}
$$

which is identical to [10]. In other words, the solution of the equivalent systems [2], [4], and [7] can be achieved by iterating between system [9] and [10], where [9] can be viewed as the regular MME used for most classical genetic evaluations, except for a correction term $\alpha_{u} \mathbf{A}_{22}^{-1} \hat{\mathbf{d}}_{2}=\alpha_{u}(\hat{\boldsymbol{\varphi}}-\hat{\boldsymbol{\gamma}})$ of the right-hand side for genotyped animals. This contribution of genomic information is proportional to the difference between 2 similar expressions: $\hat{\boldsymbol{\varphi}}=\mathbf{A}_{22}^{-1} \hat{\mathbf{u}}_{2}$ and $\hat{\boldsymbol{\gamma}}=\mathbf{G}^{-1} \hat{\mathbf{u}}_{2}$. If $\mathbf{A}_{22}$ and $\mathbf{G}$ are of large size, an iterative algorithm to solve $\mathbf{A}_{22} \hat{\boldsymbol{\varphi}}=\hat{\mathbf{u}}_{2}$ and $\mathbf{G} \hat{\gamma}=\hat{\mathbf{u}}_{2}$ can be implemented.

It is well known (Coleman, 1984) that convergence of (block) Gauss-Seidel or SOR for $\mathbf{B x}=\mathbf{t}$, where $\mathbf{B}$ is the matrix of coefficients of the MME and $\mathbf{x}$ is the vector of unknowns at each iteration, does exist if and only if $\mathbf{B}$ is positive definite when $\mathbf{B}$ is symmetric and, therefore, $[9-10]$ will converge if [7] is positive definite. It can be shown that this is the case if $\mathbf{G}-\mathbf{A}_{22}$ is itself positive definite. This is rarely the case.

However, a less-known result is that, for $\mathbf{B}$ not symmetric, if $\mathbf{B}+\mathbf{B}^{\prime}$ is positive definite (this is the case of [4]) then, a positive, real number $w_{g}$ exists so that for 0 $<w<w_{g}$, the iterative solution using SOR/SUR with a relaxation parameter $w$ will converge to the solution (Broyden, 1964; Varga and Niethammer, 1993). Usually $w_{g}$ will be lower than 1 . Therefore, because $\mathbf{G}-\mathbf{A}_{22}$ is usually not positive definite, updates of iterative SSGBLUP using [9-10], need to be done by block SUR (Broyden, 1964), where new solutions are a weighted average of the solutions to [9-10] and the former solutions. Therefore, SUR iterations should be as follows. At iteration $t$,

$$
\left[\begin{array}{c}
\hat{\mathbf{b}} \\
\hat{\mathbf{u}}_{1} \\
\hat{\mathbf{u}}_{2}
\end{array}\right]^{t}=\omega\left[\begin{array}{c}
\hat{\mathbf{b}}^{*} \\
\hat{\mathbf{u}}_{1} \\
\hat{\mathbf{u}}_{2}
\end{array}\right]^{*}+(1-\omega)\left[\begin{array}{c}
\hat{\mathbf{b}} \\
\hat{\mathbf{u}}_{1} \\
\hat{\mathbf{u}}_{2}
\end{array}\right]^{-1},
$$




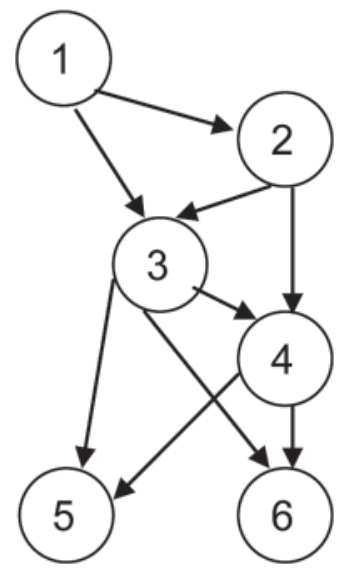

Figure 1. Example pedigree.

where $\left[\begin{array}{c}\hat{\mathbf{b}} \\ \hat{\mathbf{u}}_{1} \\ \hat{\mathbf{u}}_{2}\end{array}\right]^{*}$ is a solution to $[9], \hat{\varphi}^{t}=\omega \hat{\varphi}^{*}+(1-\omega) \hat{\varphi}^{t-1}$, and $\hat{\gamma}^{t}=\omega \hat{\gamma}^{*}+(1-\omega) \hat{\gamma}^{t-1}$, where $\hat{\varphi}^{*}$ and $\hat{\gamma}^{*}$ are solutions to [10]. As for the system of Equation 5, this can be implemented iteratively as follows:

(1) Solve

$$
\begin{gathered}
{\left[\begin{array}{ccc}
\mathbf{X}^{\prime} \mathbf{X} & \mathbf{X}_{1}^{\prime} \mathbf{W}_{1} & \mathbf{X}_{2}^{\prime} \mathbf{W}_{2} \\
\mathbf{W}_{1}^{\prime} \mathbf{X}_{1} & \mathbf{W}_{1}^{\prime} \mathbf{W}_{1}+\alpha_{u} \mathbf{A}^{11} & \alpha_{u} \mathbf{A}^{12} \\
\mathbf{W}_{2}^{\prime} \mathbf{X}_{2} & \alpha_{u} \mathbf{A}^{21} & \mathbf{W}_{2}^{\prime} \mathbf{W}_{2}+\alpha_{u} \mathbf{A}^{22}
\end{array}\right]\left[\begin{array}{c}
\hat{\mathbf{b}} \\
\hat{\mathbf{u}}_{1} \\
\hat{\mathbf{u}}_{2}
\end{array}\right]=} \\
{\left[\begin{array}{c}
\mathbf{X}^{\prime} \mathbf{y} \\
\mathbf{W}_{1}^{\prime} \mathbf{y}_{1} \\
\mathbf{W}_{2}^{\prime} \mathbf{y}_{2}
\end{array}\right]+\left[\begin{array}{c}
\mathbf{0} \\
\mathbf{0} \\
\alpha_{u} \hat{\boldsymbol{\varphi}}-\alpha_{u} \hat{\gamma}
\end{array}\right] ;}
\end{gathered}
$$

(2) Solve $\mathbf{A}_{22} \hat{\boldsymbol{\varphi}}=\hat{\mathbf{u}}_{2}$ for $\hat{\boldsymbol{\varphi}}$;

(3) Either solve $\hat{\mathbf{g}}=\mathbf{D} \mathbf{Z}^{\prime} \mathbf{G}^{-1} \hat{\mathbf{u}}_{2}$ and $\hat{\boldsymbol{\gamma}}=\mathbf{G}^{-1} \mathbf{Z} \hat{\mathbf{g}}$ or compute $\hat{\boldsymbol{\gamma}}=\mathbf{G}^{-1} \hat{\mathbf{u}}_{2}$ and $\hat{\mathbf{g}}=\mathbf{D} \mathbf{Z}^{\prime} \hat{\boldsymbol{\gamma}}$. [13]

\section{Small Example}

Consider the pedigree in Figure 1. Only 2 individuals in this pedigree are genotyped: 4 and 6 . We simulated by gene dropping 20 biallelic markers. As a result, matrices $\mathbf{G}$ and $\mathbf{A}_{22}$ have values

$$
\mathbf{G}=\left(\begin{array}{ll}
1.36 & 1.45 \\
1.45 & 2.45
\end{array}\right)
$$

and

$$
\mathbf{A}_{22}=\left(\begin{array}{ll}
1.38 & 1.19 \\
1.19 & 1.50
\end{array}\right)
$$

records are $\mathbf{y}=(1,2,0.3,5,0.2,0.1)$, and the heritability is $\mathrm{h}^{2}=0.1$; the left-hand side of the mixed model Equation [4] is shown in Figure 2 to show the sparsity pattern. Equation [5] and regular SSGBLUP [3], unsymmetric extended SSGBLUP [4], iterative SSGBLUP [9-10] (with $\omega=1$ ) provided exactly the same results, namely $\hat{\mathbf{u}}=(-0.12,0.00,-0.17,0.03,-0.11,-0.19)$ with $\hat{\mathbf{d}}_{2}=(-0.07,-0.16)$. This contrasts with $\hat{\mathbf{u}}=$ $(-0.05,0.05,-0.07,0.10,-0.03,-0.03)$ when the genomic information is ignored.

\section{Simulated Example}

We used the QMSim simulator (Sargolzaei and Schenkel, 2009) to simulate 28,800 individuals, from which 1,800 were genotyped for 30,000 polymorphic loci. The scenario is roughly as in Vitezica et al. (2011). Genotyped individuals were the last 2 generations, half of them with performance records and half without. Matrix $\mathbf{G}$ was created and tuned to fit $\mathbf{A}_{22}$ as in Vitezica et

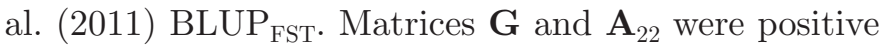
definite; $\mathbf{G}-\mathbf{A}_{22}$ was not: about half of its eigenvalues were negative.

Three ways of estimating breeding values were used: regular SSGBLUP [3], unsymmetric extended SSGBLUP [4], and iterative SSGBLUP [9-10]. In regular SSGBLUP, $\mathbf{G}$ and $\mathbf{A}_{22}$ and their inverses were created using preGSf90 software (Aguilar et al., 2011); resolution was by preconditioned conjugate gradient (PCG) as implemented in the Blupf90 package. In unsymmetric extended SSGBLUP, $\mathbf{G}$ and $\mathbf{A}_{22}$ were read from file and stored in memory; resolution was by BiCGstab as implemented by I. Aguilar (INIA Las Brujas, Canelones, Uruguay) in the Blupf90 package. In iterative SSGBLUP, 2 solution loops were used: the outer loops updated the right-hand side in [9] and [10]; the inner loops solved [9] and [10]. Equation 9 was solved by PCG; as for [10], 2 strategies were used: one was to solve $\mathbf{A}_{22} \hat{\boldsymbol{\varphi}}=\hat{\mathbf{u}}_{2}$ and $\mathbf{G} \hat{\boldsymbol{\gamma}}=\hat{\mathbf{u}}_{2}$; this mimics the iteration on data situation. The other one was to apply (17) using $\hat{\boldsymbol{\varphi}}=\mathbf{A}_{22}^{-1} \hat{\mathbf{u}}_{2}$ and $\hat{\boldsymbol{\gamma}}=\mathbf{G}^{-1} \hat{\mathbf{u}}_{2}$ with already inverted $\mathbf{G}^{-1}$ and $\mathbf{A}_{22}^{-1}$.

Values of $\omega=(0.2,0.5,0.6,0.7,1.0)$ were tried. In all cases, equations were stored in memory using hash techniques, but conclusions about convergence are the same as for iteration on data techniques. The number of nonzero elements in traditional MME was 214,911. The number of nonzero elements in $\mathbf{G}$ and $\mathbf{A}_{22}$ was $1,800^{2}$ $=3,240,000$. Convergence levels were set to $10^{-12}$ in all 
STRATEGIES FOR SINGLE-STEP GENOMIC EVALUATION

\begin{tabular}{|c|c|c|c|c|c|c|c|c|c|c|c|}
\hline & {$[, 1]$} & {$[, 2]$} & {$[, 3]$} & {$[, 4]$} & {$[, 5]$} & {$[, 6]$} & {$[, 7]$} & {$[, 8]$} & {$[, 9]$} & {$[, 10]$} & {$[, 11]$} \\
\hline$[1]$, & 6.00 & 1.0 & 1.00 & 1.00 & 1.00 & 1.00 & 1.00 & & & & \\
\hline$[2]$, & 1.00 & 17.5 & -1.50 & -9.00 & & & & & & & \\
\hline$[3]$, & 1.00 & -1.5 & 22.64 & -3.86 & -10.29 & & & & & & \\
\hline$[4]$, & 1.00 & -9.0 & -3.86 & 37.23 & 2.81 & -13.09 & -13.09 & & & & \\
\hline$[5]$, & 1.00 & & -10.29 & 2.81 & 34.66 & -13.09 & -13.09 & 9.00 & & -9.00 & \\
\hline$[6]$, & 1.00 & & & -13.09 & -13.09 & 27.18 & & & & & \\
\hline$[7]$, & 1.00 & & & -13.09 & -13.09 & & 27.18 & & 9.00 & & -9.00 \\
\hline$[8]$, & & & & & 9.00 & & & 12.38 & 10.69 & & \\
\hline$[9]$, & & & & & & & 9.00 & 10.69 & 13.50 & & \\
\hline$[10]$, & & & & & 9.00 & & & & & 12.24 & 13.03 \\
\hline$[11]$, & & & & & & & 9.00 & & & 13.03 & 22.10 \\
\hline
\end{tabular}

Figure 2. Left-hand side of the mixed model equations according to Equation 4 for the example.

iterative solvers (PCG, BiCGstab, and SUR), although they are not strictly comparable across methods. Table 1 summarizes the different computing options.

\section{RESULTS AND DISCUSSION}

\section{Simulated Example}

Table 1 reflects the computing times and number of operations for each method. Figure 3 shows the progression of convergences for each method. All methods reached the same solutions (correlation $>0.9999$ ) once converged. The iterative SSGBLUP did not converge for $\omega=0.6$ or higher, whereas convergence for $\omega=0.5$ took 83 iterations (213 iterations for $\omega=0.2$ ). Overall, and according to results in Table 1, all solvers are competitive. However, this will not be so for other numbers of total and genotyped individuals and number of SNP. We will discuss this point later.

The need for integrating all data in genomic evaluation implementations is well acknowledged (VanRaden et al., 2009; Wolc et al., 2011). In addition to being theoretically appealing, implementation of the single-step methodology provides good results in both simulated (Christensen and Lund, 2010; Vitezica et al., 2011) and dairy cattle, poultry, and pig real data (Aguilar et al., 2010; Chen et al., 2011; Christensen et al., 2011). It is crucial to use single step to get unbiased estimates if early selection based on genomic proofs becomes a reality (Patry and Ducrocq, 2011a). Yet, the need for new software as well as a lack of understanding of features of the method seem to somehow hamper its implemen- tation. We herein provide an alternate derivation and techniques (expansion of MME, iterative solutions, and algorithms that can work for iteration on data) that may help practitioners. The expansion was used by Henderson (1977) to include the sparse inverse of the numerator relationship matrix in the MME. The alternate derivation follows the logic of unknown parent groups (e.g., Quaas and Pollak, 1981), with the added complexity that, in the latter, group effects were added to the founders, whereas here deviations $\mathbf{d}$ due to SNP effects are added "in the middle" of the pedigree. We prove in Appendix $\mathrm{C}$ that this derivation is correct regardless of whether $\mathrm{G}-\mathrm{A}_{22}$ is positive definite or not.

\section{Distributional Properties}

In all symmetric transformations, the distributional properties of [1] are kept. For instance, reliabilities of $\hat{\mathbf{u}}$ can be computed from the inverse of the left-hand side of [4] (in its symmetric counterpart) or [5] or [7]. Further, a Gibbs sampler can be implemented by using [9-10] or [9, 10, and 13]. This Gibbs sampler can eventually update $\mathbf{G}$ without the need to calculate its inverse.

\section{Theoretical Convergence}

As presented above, let $\mathbf{B}$ be the left-hand side coefficients of [4]. Because $\mathbf{B}+\mathbf{B}^{\prime}$ is positive definite, then for some $0<w<w_{g}$, the iterative solution using SUR will converge to the solution (Broyden, 1964; Varga and 
Table 1. Computing options and performances to solve the single-step model in a simulated example

\begin{tabular}{lcccc}
\hline & $\begin{array}{c}\text { Regular } \\
\text { Item }^{1}\end{array}$ & $\begin{array}{c}\text { Unsymmetric } \\
\text { SSGBLUP }\end{array}$ & $\begin{array}{c}\text { Iterative } \\
\text { extended SSGBLUP }\end{array}$ & Iterative \\
Needs inverted $\mathbf{G}^{-1}$ and $\mathbf{A}_{22}^{-1}$ & Yes & No & No & Yes \\
Solving method & PCG $^{3}$ & BiCGstab $^{4}$ & SUR $^{5}$ (outer loop) & SUR (outer loop) \\
Number of iterations & 62 & & PCG (Equations 9 and 10) $^{6}$ & PCG (Equation 9) \\
Computing time, s & 2 & 240 & $283^{6}$ & $83^{6}$ \\
\hline
\end{tabular}

${ }^{1} \mathbf{G}=$ genomic relationship matrix; $\mathbf{A}_{22}=$ pedigree relationship matrix.

${ }^{2}$ SSGBLUP $=$ single-step genomic BLUP.

${ }^{3}$ Preconditioned conjugate gradient.

${ }^{4}$ Biconjugate gradient stabilized method.

${ }^{5}$ Successive underrelaxation.

${ }^{6}$ Outer loop.

Niethammer, 1993). Usually $w_{g}$ is lower than 1 . Therefore iterative SSGBLUP [9-10], which is a block variant of SUR (Coleman, 1984), will converge to a solution. As for the BiCGstab used in unsymmetric extended
SSGBLUP, this is a robust algorithm (van der Vorst, 2003). Note that only (block) stationary methods were considered here. Nonstationary methods (e.g., variants of PCG) could lead to faster convergence.

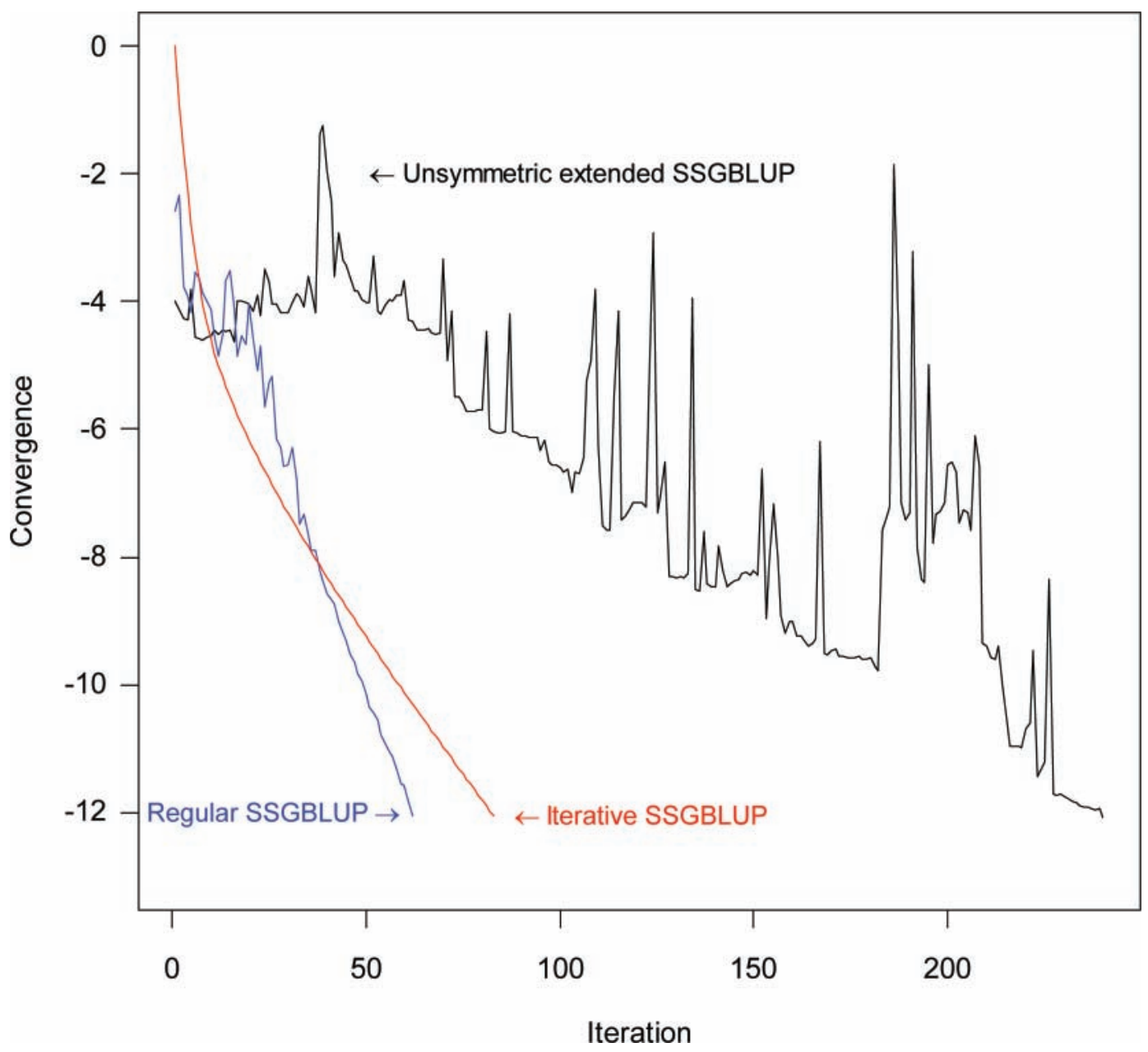

Figure 3. Convergence of regular single-step genomic BLUP (SSGBLUP), unsymmetric extended SSGBLUP, and iterative SSGBLUP in the simulated data. Color version available in the online PDF. 


\section{Different Definitions of G}

An overlooked aspect about Equation 2 is that the derivation is general: other matrices can be used instead of the VanRaden (2008) G, as long as they describe additive covariances (not necessarily relationships) among individuals. For instance, in the French dairy cattle evaluation, gametic effects based on haplotype segments are used (Guillaume et al., 2008). If the incidence matrix of gametic effects in locus i is $\mathbf{Z}_{\mathrm{i}}$, and the covariance matrix among gametic effects is $\boldsymbol{\Lambda}_{\mathrm{i}}$, then $\mathbf{G}=\kappa_{0} \mathbf{A}_{22}+\Sigma \kappa_{i} \mathbf{Z}_{i} \boldsymbol{\Lambda}_{i} \mathbf{Z}_{i}{ }^{\prime}$, where $\kappa_{i}$ are fractions of the total genetic variance.

\section{Computing}

In unsymmetric extended SSGBLUP or iterative SSGBLUP, no explicit inverses of $\mathbf{A}_{22}$ and $\mathbf{G}$ are needed. These are expensive. Computation of $\mathbf{A}_{22}$ has a cost of $\mathrm{O}(p n)$ or $\mathrm{O}\left(p^{2}\right)$ if a sub-pedigree is extracted (Aguilar et al., 2011); computation of $\mathbf{G}$ has $\mathrm{O}\left(m p^{2}\right)$ cost. Inverting either matrix costs $\mathrm{O}\left(p^{3}\right)$. Direct computation of $\mathbf{G}^{-1}$ costs $\mathrm{O}\left(m p^{2}\right)$ if the Sherman and Morrison (1949) formula is used. This is not tenable for very large $p$.

However, in such a case, equations can be solved by iteration on data using Jacobi or PCG methods, without explicit computation or storage of either $\mathbf{A}_{22}$ or $\mathbf{G}$. In both, the kernel and more expensive part of the solving method consists in repeated multiplications of the form $\mathbf{t}=\mathbf{B} \mathbf{x}$ (in BiCGstab, this computation is done twice per round). For classical MME (i.e., [9]), the PCG method using iteration on data has been described by Strandén and Lidauer (1999), and consists in reading data and pedigree files and adding contributions. Modifications for unsymmetric extended SSGBLUP [4] would consist of adding the $\mathbf{A}_{22} \boldsymbol{\varphi}$ and $\mathbf{G} \gamma$ contributions. The first one can be done, without explicit computing of $\mathbf{A}_{22}$, by reading twice the pedigree file, with cost $\mathrm{O}(n)$ (Misztal et al., 2009). The second one can be done, if $\mathbf{G}$ has a form similar to $\mathbf{G}=\mathbf{Z D Z}^{\prime}$ (e.g., Guillaume et al., 2008; VanRaden, 2008; Calus et al., 2009) by computing $\mathbf{G} \boldsymbol{\gamma}=\mathbf{Z}\left[\mathbf{D}\left(\mathbf{Z}^{\prime} \boldsymbol{\gamma}\right)\right]$ (Strandén and Garrick, $2009)$, at a cost $\mathrm{O}(m p)$ if $\mathbf{D}$ is diagonal or very sparse, which is usually the case. If $m p$ is very large, the product can be computed by iteration on data. In iterative SSGBLUP, solving $\mathbf{G} \hat{\gamma}=\hat{\mathbf{u}}_{2}$ and $\mathbf{A}_{22} \hat{\boldsymbol{\varphi}}=\hat{\mathbf{u}}_{2}$ (i.e., [10]) can be achieved by PCG with the same requirements. Indeed, $\mathrm{O}(\mathrm{mp})$ is the lowest bound attainable, because this is the cost of reading the raw genotypic data.

In regular SSGBLUP equations (i.e., [3]), inversions of $\mathbf{G}$ and $\mathbf{A}_{22}$ can be avoided as well. The multiplication $\mathbf{t}=\mathbf{B} \mathbf{x}$, needed in PCG:

$$
\left[\begin{array}{ccc}
\mathbf{X}^{\prime} \mathbf{X} & \mathbf{X}_{1}^{\prime} \mathbf{W}_{1} & \mathbf{X}_{2}^{\prime} \mathbf{W}_{2} \\
\mathbf{W}_{1}^{\prime} \mathbf{X}_{1} & \mathbf{W}_{1}^{\prime} \mathbf{W}_{1}+\alpha_{u} \mathbf{A}^{11} & \alpha_{u} \mathbf{A}^{12} \\
\mathbf{W}_{2}^{\prime} \mathbf{X}_{2} & \alpha_{u} \mathbf{A}^{12} & \mathbf{W}_{2}^{\prime} \mathbf{W}_{2}+\alpha_{u} \mathbf{A}^{22}+\alpha_{u}\left(\mathbf{G}^{-1}-\mathbf{A}_{22}^{-1}\right)
\end{array}\right]\left[\begin{array}{c}
\hat{\mathbf{b}} \\
\hat{\mathbf{u}}_{1} \\
\hat{\mathbf{u}}_{2}
\end{array}\right]
$$

can be obtained by summing

$$
\left[\begin{array}{ccc}
\mathbf{X}^{\prime} \mathbf{X} & \mathbf{X}_{1}^{\prime} \mathbf{W}_{1} & \mathbf{X}_{2}^{\prime} \mathbf{W}_{2} \\
\mathbf{W}_{1}^{\prime} \mathbf{X}_{1} & \mathbf{W}_{1}^{\prime} \mathbf{W}_{1}+\alpha_{u} \mathbf{A}^{11} & \alpha_{u} \mathbf{A}^{12} \\
\mathbf{W}_{2}^{\prime} \mathbf{X}_{2} & \alpha_{u} \mathbf{A}^{12} & \mathbf{W}_{2}^{\prime} \mathbf{W}_{2}+\alpha_{u} \mathbf{A}^{22}
\end{array}\right]\left[\begin{array}{c}
\hat{\mathbf{b}} \\
\hat{\mathbf{u}}_{1} \\
\hat{\mathbf{u}}_{2}
\end{array}\right]
$$

and

$$
\alpha_{u}\left(\mathbf{G}^{-1} \hat{\mathbf{u}}_{2}-\mathbf{A}_{22}^{-1} \hat{\mathbf{u}}_{2}\right),
$$

where the latter can be constructed by solving the systems of equations $\mathbf{G} \hat{\gamma}=\hat{\mathbf{u}}_{2}$ and $\mathbf{A}_{22} \hat{\boldsymbol{\varphi}}=\hat{\mathbf{u}}_{2}$.

Therefore, solving the regular SSGBLUP Equation [3], the unsymmetric expanded SSGBLUP using [4] or [5], or the iterative SSGBLUP system [9-10] has a cost $\mathrm{O}(n)$ for the least squares, $\mathbf{A}^{-1}$, and $\mathbf{A}_{22}$ parts; and $\mathrm{O}(m p)$ for the $\mathbf{G}$ part (times a certain number of iterations to solve the equations $\mathbf{G} \hat{\boldsymbol{\gamma}}=\hat{\mathbf{u}}_{2}$ and $\mathbf{A}_{22} \hat{\boldsymbol{\varphi}}=\hat{\mathbf{u}}_{2}$, when needed). This is equivalent to the cost of running a 2-step genetic evaluation where pedigree-based and genomic-based genetic evaluations are run in succession. In addition, solving of regular MME (as in [9]) can be done using any existing, already optimized, software. Table 2 summarizes the number of operations and memory needed for each system in the cases of iteration on data. The case of regular SSGBLUP without inversion of $\mathbf{G}$ and $\mathbf{A}_{22}$ is as iterative SSGBLUP [1]. It can be seen that, whereas all methods are in principle feasible, its effectiveness for large $p$ depends critically on the number of iterations needed to achieve convergence of global equations and of blocks in the case of iterative SSGBLUP and of regular SSGBLUP without inversion. This deserves further investigation with real, large data sets.

\section{Estimation of SNP Effects}

As for Equation 5, this is explicit in SNP effects. Note that $\hat{\mathbf{g}}=\mathbf{D Z}^{\prime} \mathbf{G}^{-1} \hat{\mathbf{u}}_{2}$; that is, assuming multivariate normality, $\hat{\mathbf{g}}$ is the best predictor of $\mathbf{g}$, given $\hat{\mathbf{u}}_{2}$, or, equivalently, the solution to the system $\hat{\mathbf{u}}_{2}=\mathbf{Z g}$, with $\mathbf{D}$ the covariance matrix of $\mathbf{g}$ and no residual terms. There are ways of solving for $\mathbf{g}$, the marker effects, which do not involve explicit construction or inversion 
of G (Legarra and Misztal, 2008; VanRaden, 2008; Strandén and Garrick, 2009) and also nonlinear methods (BayesB and Bayesian Lasso, among others; Meuwissen et al., 2001; VanRaden, 2008; de los Campos et al., 2009). Note that even if there is no residual $\left(\hat{\mathbf{u}}_{2}\right.$ $=\mathbf{Z g}$ ), $\mathbf{g}$ has a posterior distribution because $\mathbf{g}$ has a prior distribution; therefore, the different $\mathbf{g}$ where $\hat{\mathbf{u}}_{2}=$ Zg (and, therefore, equally likely) have different a posteriori probabilities. Thus, the same software used for nonlinear genomic evaluation methods can be used for single step, provided it iteratively uses current values of $\hat{\mathbf{u}}_{2}$ as input data, possibly (for the sake of practicality) fitting a very small residual variance. Use of nonlinear models for $\mathbf{g}$ within [5] will be an approximation because [5] was derived on the pretence of multivariate normality. Matrix $\mathbf{G}$ involved in computation of $\gamma=$ $\mathbf{G}^{-1} \mathbf{Z g}$ should be updated if the variance explained by each SNP changes (as in BayesA, BayesB, or the Bayesian Lasso). Whereas individual variances of SNP effects depend much on the prior assumptions, use of estimated $\mathbf{G}$, which is a function of all of them, seems to be a robust alternative (Zhang et al., 2010; Legarra et al., 2011), possibly because the weight of the prior fades out.

\section{A Special Case}

Situations exist where it is desired to base genomic evaluations on a reference population for genomic selection differing from the complete list of genotyped animals in the national evaluation. This is the case when there are some phenotypes from genotyped animals that we would like to exclude from the genomic evaluation part: in most European countries and Canada, own records from bull dams are excluded for the estimation of marker effects, because of fear of preferential treatment. Even if cows are not subject to preferential treat- ment, the bias in genotyping (genotyped animals having higher breeding values than the average) might lead to bias of genomic proofs (Vitezica et al., 2011). On the other hand, there may also be some extra phenotypes from genotyped animals that need to be included in the genomic evaluation part but are not routinely included in regular evaluations. A typical example is phenotypes (usually deregressed international EBV) from foreign bulls in multinational reference populations. A variant of the single-step approach would consist of including a usual genomic evaluation before the second step (i.e., Equation 10) of the iterative procedure to get a $\hat{\mathbf{u}}_{2}$ estimate based on the desired information. This estimate would be plugged into [10]. The convergence in practice to a proper solution needs to be studied.

\section{Current Limits}

In practice, to our knowledge, current software (as implemented by Aguilar et al., 2011) and hardware has allowed the use of single step with computed inverses of $\mathbf{G}$ and $\mathbf{A}_{22}$ for up to 17,000 genotyped individuals and 18 traits (Tsuruta et al., 2011). This is possibly enough for many animal populations (e.g., in other dairy cattle or sheep populations). However, in the Holstein population of large countries (United States, Germany, and France, among others), the number of genotyped animals is already or will soon exceed 50,000 or 100,000 animals. Therefore, at some point, explicit inverses will no longer be possible to be computed and stored. Also, with high-density SNP chips, computation of $\mathbf{G}$ itself will become a challenge; again, using approaches that do not compute $\mathbf{G}$ explicitly, (e.g., solving [9-10] or [4] by BiCGstab) the cost is $\mathrm{O}(m p)$, that is, the same order of magnitude as reading the data. Further, iteration on data are available so memory is not a real challenge. Extensive testing in challenging situations (complex

Table 2. Number of operations and memory needed for iteration on data with different solvers ${ }^{1}$

\begin{tabular}{|c|c|c|c|c|}
\hline Item $^{2}$ & $\begin{array}{l}\text { Regular } \\
\text { SSGBLUP }\end{array}$ & $\begin{array}{l}\text { Unsymmetric } \\
\text { extended } \\
\text { SSGBLUP }\end{array}$ & $\begin{array}{l}\text { Iterative } \\
\text { SSGBLUP [1] }\end{array}$ & $\begin{array}{l}\text { Iterative } \\
\text { SSGBLUP [2] }\end{array}$ \\
\hline $\begin{array}{l}\mathbf{G}^{-1} \text { and } \mathbf{A}_{22}^{-1} \text { needed } \\
\text { Computing } \mathbf{G}^{-1} \text { and } \mathbf{A}_{22}^{-1} \\
\text { Solving ( } 1 \text { iteration) }\end{array}$ & $\begin{array}{l}\text { Yes } \\
\mathrm{O}\left(m p^{2}\right)+\mathrm{O}(p n)+ \\
\mathrm{O}\left(p^{3}\right)+\mathrm{O}\left(p^{3}\right) \\
\mathrm{O}\left(p^{2}+n\right) \\
\mathrm{O}\left(p^{2}+n\right)\end{array}$ & $\begin{array}{l}\text { No. of operations } \\
\text { No } \\
0 \\
\mathrm{O}(p m+n+n) \\
\text { Memory } \\
\mathrm{O}(n+2 p)\end{array}$ & $\begin{array}{l}\text { No } \\
0 \\
\mathrm{O}\left(t_{M} n+t_{G} p m+t_{A} n\right) \\
\mathrm{O}(n+2 p)\end{array}$ & $\begin{array}{l}\text { Yes } \\
\mathrm{O}\left(m p^{2}\right)+\mathrm{O}(p n)+ \\
\mathrm{O}\left(p^{3}\right)+\mathrm{O}\left(p^{3}\right) \\
\mathrm{O}\left(t_{M} n+2 p^{2}\right) \\
\mathrm{O}\left(p^{2}+n+2 p\right)\end{array}$ \\
\hline
\end{tabular}

${ }^{1} m=$ number of SNP; $\mathrm{n}=$ number of individuals in pedigree; $p=$ number of genotyped individuals; $t_{M}, t_{G}$, and $t_{A}$ : number of iterations needed to achieve convergence of regular genetic evaluation (Equation 9), $\mathbf{G} \hat{\gamma}=\hat{\mathbf{u}}_{2}$, and $\mathbf{A}_{22} \boldsymbol{\varphi}=\hat{\mathbf{u}}_{2}$ (Equation 10), respectively.

${ }^{2} \mathbf{G}=$ genomic relationship matrix; $\mathbf{A}_{22}=$ pedigree relationship matrix.

${ }^{3}$ SSGBLUP $=$ single-step genomic BLUP. The case of regular SSGBLUP without inversion of $\mathbf{G}$ and $\mathbf{A}_{22}$ is as iterative SSGBLUP [1] without inversion. 
models and large number of genotypes) will be needed to find out the feasibility and accuracy of the solutions we propose in this work.

\section{CONCLUSIONS}

Single-step evaluations including records, pedigree, and genotypes can, in principle, be carried out with little additional computing costs or changes compared with 2-step procedures. Convergence of the method is guaranteed from existing theory. This will facilitate its introduction in routine evaluation.

\section{ACKNOWLEDGMENTS}

The authors thank the French National Research Agency (ANR, Paris, France) project AMASGEN and APISGENE for financing. A. Legarra thanks the European FEDER funds for project GENOMIA through POCTEFA (http://www.poctefa.eu). Ignacy Misztal (University of Georgia, Athens), Ignacio Aguilar (INIA, Las Brujas, Uruguay), Gregor Gorjanc (University of Ljubljana, Ljubljana, Slovenia), Paul VanRaden (USDA, Beltsville, MD), Dave Johnson (LIC, Hamilton, New Zealand), and Daniel Gianola (University of Madison, WI) are thanked for fruitful comments. Use of code by Ignacio Aguilar for the BiCGstab solver and the unsymmetric storage for the Blupf90 software is particularly acknowledged. The authors acknowledge the editor and reviewers for suggestions and requirements largely improving the final manuscript. This project was partly supported by the Toulouse bioinformatics platform (France; http://bioinfo.genotoul.fr/).

\section{REFERENCES}

Aguilar, I., I. Misztal, D. L. Johnson, A. Legarra, S. Tsuruta, and T. J. Lawlor. 2010. Hot topic: A unified approach to utilize phenotypic, full pedigree, and genomic information for genetic evaluation of Holstein final score. J. Dairy Sci. 93:743-752.

Aguilar, I., I. Misztal, A. Legarra, and S. Tsuruta. 2011. Efficient computations of genomic relationship matrix and other matrices used in the single-step evaluation. J. Anim. Breed. Genet. 128:422-428.

Besbes, B., V. Ducrocq, J. Foulley, M. Protais, A. Tavernier, M. Tixier-Boichard, and C. Beaumont. 1992. Estimation of genetic parameters of egg production traits of laying hens by restricted maximum likelihood applied to a multiple-trait reduced animal model. Genet. Sel. Evol. 24:1-14.

Broyden, C. 1964. On convergence criteria for the method of successive over-relaxation. Math. Comput. 18:136-141.

Calus, M. P. L., T. H. E. Meuwissen, J. J. Windig, E. F. Knol, C. Schrooten, A. L. J. Vereijken, and R. F. Veerkamp. 2009. Effects of the number of markers per haplotype and clustering of haplotypes on the accuracy of QTL mapping and prediction of genomic breeding values. Genet. Sel. Evol. 41:11.

Chen, C. Y., I. Misztal, I. Aguilar, A. Legarra, and W. M. Muir. 2011. Effect of different genomic relationship matrices on accuracy and scale. J. Anim. Sci. 89:2673-2679.
Christensen, O. F., and M. S. Lund. 2010. Genomic prediction when some animals are not genotyped. Genet. Sel. Evol. 42:2.

Christensen, O. F., P. Madsen, B. Nielsen, T. Ostersen, and G. Su. 2011. Genomic predictions on pigs using the single step method. 62nd Annu. Meet. European Fed. Anim. Sci., Stavanger, Norway. Book of Abstracts, 17: 393. Wageningen Academic Publishers, Wageningen, the Netherlands.

Cockerham, C. C. 1969. Variance of gene frequencies. Evolution $23: 72-84$

Coleman, T. F. 1984. Large Sparse Numerical Optimization. SpringerVerlag New York Inc., New York, NY.

de los Campos, G., H. Naya, D. Gianola, J. Crossa, A. Legarra, E. Manfredi, K. Weigel, and J. M. Cotes. 2009. Predicting quantitative traits with regression models for dense molecular markers and pedigree. Genetics 182:375-385.

Friedman, J., T. Hastie, and R. Tibshirani. 2010. Regularization paths for generalized linear models via coordinate descent. J. Stat. Softw. 33:1-22.

Gengler, N., P. Mayeres, and M. Szydlowski. 2007. A simple method to approximate gene content in large pedigree populations: Application to the myostatin gene in dual-purpose Belgian Blue cattle. Animal 1:21-28.

Guillaume, F., S. Fritz, D. Boichard, and T. Druet. 2008. Short communication: Correlations of marker-assisted breeding values with progeny-test breeding values for eight hundred ninety-nine French Holstein bulls. J. Dairy Sci. 91:2520-2522.

Henderson, C. 1973. Sire evaluation and genetic trends. Pages 10-41 in Proc. Anim. Breed. Genet. Symp. in Honor of J. L. Lush. Am. Soc. Anim. Sci. and Am. Dairy Sci. Assoc., Champaign, IL.

Henderson, C. 1977. Best linear unbiased prediction of breeding values not in the model for records. J. Dairy Sci. 60:783-787.

Henderson, C. R. 1976. A simple method for computing the inverse of a numerator relationship matrix used in prediction of breeding values. Biometrics 32:69-83.

Legarra, A., I. Aguilar, and I. Misztal. 2009. A relationship matrix including full pedigree and genomic information. J. Dairy Sci. 92:4656-4663.

Legarra, A., and I. Misztal. 2008. Technical note: Computing strategies in genome-wide selection. J. Dairy Sci. 91:360-366.

Legarra, A., C. Robert-Granié, P. Croiseau, F. Guillaume, and S. Fritz. 2011. Improved Lasso for genomic selection. Genet. Res. (Camb.) 93:77-87.

Mäntysaari, E., and I. Strandén. 2010. Use of bivariate EBV-DGV model to combine genomic and conventional breeding value evaluations. Comm. 353 in Proc. 9th World Congr. Genet. Appl. Livest. Prod., Leipzig, Germany. German Society for Animal Science, Giessen, Germany.

Meuwissen, T. H. E., B. J. Hayes, and M. E. Goddard. 2001. Prediction of total genetic value using genome-wide dense marker maps. Genetics 157:1819-1829.

Misztal, I., and D. Gianola. 1987. Indirect solution of mixed model equations. J. Dairy Sci. 70:716-723.

Misztal, I., A. Legarra, and I. Aguilar. 2009. Computing procedures for genetic evaluation including phenotypic, full pedigree, and genomic information. J. Dairy Sci. 92:4648-4655.

Patry, C., and V. Ducrocq. 2011a. Evidence of biases in genetic evaluations due to genomic preselection in dairy cattle. J. Dairy Sci. 94:1011-1020.

Patry, C., and V. Ducrocq. 2011b. Accounting for genomic pre-selection in national genetic dairy cattle evaluations. Genet. Sel. Evol. 43:30.

Picinbono, B. 1996. Second-order complex random vectors and normal distributions. IEEE Trans. Signal Processing 44:2637-2640.

Quaas, R., and E. Pollak. 1981. Modified equations for sire models with groups. J. Dairy Sci. 64:1868-1872.

Sargolzaei, M., and F. S. Schenkel. 2009. QMSim: A large-scale genome simulator for livestock. Bioinformatics 25:680-681.

Schaeffer, L., and B. Kennedy. 1986. Computing strategies for solving mixed model equations. J. Dairy Sci. 69:575-579.

Searle, S. R. 1982. Matrix Algebra Useful for Statistics. John Wiley, New York, NY. 
Shepherd, R. K., T. H. E. Meuwissen, and J. A. Woolliams. 2010. Genomic selection and complex trait prediction using a fast EM algorithm applied to genome-wide markers. BMC Bioinformatics 11:529.

Sherman, J., and W. J. Morrison. 1949. Adjustment of an inverse matrix corresponding to changes in the elements of a given column or a given row of the original matrix. Ann. Math. Stat. 20:621 (Abstr.)

Strandén, I., and D. J. Garrick. 2009. Technical note: Derivation of equivalent computing algorithms for genomic predictions and reliabilities of animal merit. J. Dairy Sci. 92:2971-2975.

Strandén, I., and M. Lidauer. 1999. Solving large mixed linear models using preconditioned conjugate gradient iteration. J. Dairy Sci. $82: 2779-2787$

Thompson, R. 1979. Sire evaluation. Biometrics 35:339-353.

Thompson, R., and K. Meyer. 1990. Estimating genetic parameters using an animal model with imaginary effects. Genet. Sel. Evol. $22: 133-147$.

Tsuruta, S., I. Misztal, I. Aguilar, and T. Lawlor. 2011. Multiple-trait genomic evaluation of linear type traits using genomic and phenotypic data in US Holsteins. J. Dairy Sci. 94:4198-4204.

van der Vorst, H. A. 2003. Iterative Krylov Methods for Large Linear Systems. Vol. 13. Cambridge Univ. Press, Cambridge, UK.

VanRaden, P. M. 2008. Efficient methods to compute genomic predictions. J. Dairy Sci. 91:4414-4423.
VanRaden, P. M., C. P. V. Tassell, G. R. Wiggans, T. S. Sonstegard, R. D. Schnabel, J. F. Taylor, and F. S. Schenkel. 2009. Invited review: Reliability of genomic predictions for North American Holstein bulls. J. Dairy Sci. 92:16-24.

VanRaden, P. M., and G. R. Wiggans. 1991. Derivation, calculation, and use of national animal model information. J. Dairy Sci $74: 2737-2746$.

Varga, R. S., and W. Niethammer. 1993. A note on a perturbation analysis of iterative methods, with an application to the SSOR and ADI iterative method. Pages $18-27$ in Proc. seminar Numerical Mathematics in Theory and Practice, University of West Bohemia, Pilsen, Czech Republic.

Vitezica, Z. G., I. Aguilar, I. Misztal, and A. Legarra. 2011. Bias in genomic predictions for populations under selection. Genet. Res. (Camb.) 93:357-366.

Wolc, A., C. Stricker, J. Arango, P. Settar, J. E. Fulton, N. P. O'Sullivan, R. Preisinger, D. Habier, R. Fernando, D. J. Garrick, S. J. Lamont, and J. C. M. Dekkers. 2011. Breeding value prediction for production traits in layer chickens using pedigree or genomic relationships in a reduced animal model. Genet. Sel. Evol. 43:5.

Zhang, Z., J. Liu, X. Ding, P. Bijma, D. J. de Koning, and Q. Zhang. 2010. Best linear unbiased prediction of genomic breeding values using a trait-specific marker-derived relationship matrix. PLoS ONE 5:e12648.

\section{APPENDIX A}

A possibility in SSGBLUP MME is to specifically fit marker (or gametic) effects. Assume $\mathbf{u}_{2}=\mathbf{Z g}$, where $\mathbf{g}$ are marker effects. Consider, as in Legarra et al. (2009), $p(\mathbf{g}) \sim N(\mathbf{0}, \mathbf{D})$ and $p\left(\mathbf{u}_{1} \mid \mathbf{u}_{2}\right) \sim N\left[\mathbf{A}_{12} \mathbf{A}_{22}^{-1} \mathbf{u}_{2},\left(\mathbf{A}^{11}\right)^{-1} \sigma_{u}^{2}\right]$. The joint distribution of $\mathbf{u}_{1}$ and $\mathbf{g}$ can be written as

$$
p\left(\mathbf{u}_{1}, \mathbf{g}\right)=p\left(\mathbf{u}_{1} \mid \mathbf{g}\right) p(\mathbf{g}) \propto \exp \left[-0.5\left(\mathbf{u}_{1}-\mathbf{A}_{12} \mathbf{A}_{22}^{-1} \mathbf{Z g}\right)^{\prime} \mathbf{A}^{11} \sigma_{u}^{-2}\left(\mathbf{u}_{1}-\mathbf{A}_{12} \mathbf{A}_{22}^{-1} \mathbf{Z g}\right)\right] \exp \left[-0.5 \mathbf{g}^{\prime} \mathbf{D}^{-1} \mathbf{g}\right] .
$$

After some algebra, and using $\mathbf{A}^{11} \mathbf{A}_{12} \mathbf{A}_{22}^{-1}+\mathbf{A}^{12}=\mathbf{0}$ and $\mathbf{A}^{22}+\mathbf{A}_{22}^{-1} \mathbf{A}_{21} \mathbf{A}^{11} \mathbf{A}_{12} \mathbf{A}_{22}^{-1}=\mathbf{A}_{22}^{-1}$, this can be reduced to

$$
\begin{aligned}
& \exp \left[-0.5\left(\mathbf{u}_{\mathbf{1}}-\mathbf{A}_{\mathbf{1 2}} \mathbf{A}_{\mathbf{2}}^{-1} \mathbf{Z g}\right)^{\prime} \mathbf{A}^{11} \sigma_{u}^{-2}\left(\mathbf{u}_{\mathbf{1}}-\mathbf{A}_{12} \mathbf{A}_{22}^{-1} \mathbf{Z g}\right)\right] \exp \left[-0.5 \mathbf{g}^{\prime} \mathbf{D}^{-1} \mathbf{g}\right] \\
& \left.=\exp \left[-0.5\left(\begin{array}{ll}
\mathbf{u}_{1}^{\prime} & \mathbf{g}^{\prime}
\end{array}\right)\left(\begin{array}{cc}
\mathbf{A}^{11} \sigma_{u}^{-2} & \mathbf{A}^{12} \mathbf{Z} \sigma_{u}^{-2} \\
\mathbf{Z}^{\prime} \mathbf{A}^{21} \sigma_{u}^{-2} & \mathbf{Z}^{\prime}\left(\mathbf{A}^{21} \sigma_{u}^{-2}-\mathbf{A}_{22}^{-1} \sigma_{u}^{-2}\right.
\end{array}\right) \mathbf{Z}+\mathbf{D}^{-1}\right)\left(\begin{array}{c}
\mathbf{u}_{1} \\
\mathbf{g}
\end{array}\right)\right] .
\end{aligned}
$$

This is the kernel of a normal distribution and, thus, $p\left(\mathbf{u}_{1}, \mathbf{g}\right)=N(\mathbf{0}, \mathbf{T})$, where

In fact,

$$
\mathbf{T}^{-1}=\left(\begin{array}{cc}
\mathbf{A}^{11} \sigma_{u}^{-2} & \mathbf{A}^{12} \mathbf{Z} \sigma_{u}^{-2} \\
\mathbf{Z}^{\prime} \mathbf{A}^{21} \sigma_{u}^{-2} & \mathbf{Z}^{\prime}\left(\mathbf{A}^{22} \sigma_{u}^{-2}-\mathbf{A}_{22}^{-1} \sigma_{u}^{-2}\right) \mathbf{Z}+\mathbf{D}^{-1}
\end{array}\right) .
$$

$$
\mathbf{T}^{-1}=\sigma_{u}^{-2}\left(\begin{array}{cc}
\mathbf{I} & \mathbf{0} \\
\mathbf{0} & \mathbf{Z}^{\prime}
\end{array}\right)\left(\begin{array}{cc}
\mathbf{A}^{11} & \mathbf{A}^{12} \\
\mathbf{A}^{21} & \mathbf{A}_{22}^{-1} \mathbf{A}_{21} \mathbf{A}^{11} \mathbf{A}_{12} \mathbf{A}_{22}^{-1}
\end{array}\right)\left(\begin{array}{cc}
\mathbf{I} & \mathbf{0} \\
\mathbf{0} & \mathbf{Z}
\end{array}\right)+\left(\begin{array}{cc}
\mathbf{0} & \mathbf{0} \\
\mathbf{0} & \mathbf{D}^{-1}
\end{array}\right),
$$


which shows that $\mathbf{T}$ is always positive definite. Therefore, the MME can be constructed as

$$
\left[\begin{array}{ccc}
\mathbf{X}^{\prime} \mathbf{X} & \mathbf{X}_{1}^{\prime} \mathbf{W}_{1} & \mathbf{X}_{2}^{\prime} \mathbf{W}_{2} \\
\mathbf{W}_{1}^{\prime} \mathbf{X}_{1} & \mathbf{W}_{1}^{\prime} \mathbf{W}_{1}+\alpha_{u} \mathbf{A}^{11} & \alpha_{u} \mathbf{A}^{12} \mathbf{Z} \\
\mathbf{Z}^{\prime} \mathbf{W}_{2}^{\prime} \mathbf{X}_{2} & \alpha_{u} \mathbf{Z}^{\prime} \mathbf{A}^{12} \sigma_{u}^{-2} & \mathbf{Z}^{\prime} \mathbf{W}_{2}^{\prime} \mathbf{W}_{2} \mathbf{Z}+\alpha_{u} \mathbf{Z}^{\prime}\left(\mathbf{A}^{22}-\mathbf{A}_{22}^{-1}\right) \mathbf{Z}+\mathbf{D}^{-1} \sigma_{e}^{2}
\end{array}\right]\left[\begin{array}{c}
\hat{\mathbf{b}} \\
\hat{\mathbf{u}}_{1} \\
\hat{\mathbf{g}}
\end{array}\right]=\left[\begin{array}{c}
\mathbf{X}^{\prime} \mathbf{y} \\
\mathbf{W}_{1}^{\prime} \mathbf{y}_{1} \\
\mathbf{Z}^{\prime} \mathbf{W}_{2}^{\prime} \mathbf{y}_{2}
\end{array}\right],
$$

which, by including additional unknowns $(\hat{\varphi})$ as in expression [4], can be written as

$$
\left[\begin{array}{cccc}
\mathbf{X}^{\prime} \mathbf{X} & \mathbf{X}_{1}^{\prime} \mathbf{W}_{1} & \mathbf{X}_{2}^{\prime} \mathbf{W}_{2} \mathbf{Z} & \mathbf{0} \\
\mathbf{W}_{1}^{\prime} \mathbf{X}_{1} & \mathbf{W}_{1}^{\prime} \mathbf{W}_{1}+\alpha_{u} \mathbf{A}^{11} & \alpha_{u} \mathbf{A}^{12} \mathbf{Z} & \mathbf{0} \\
\mathbf{Z}^{\prime} \mathbf{W}_{2}^{\prime} \mathbf{X}_{2} & \alpha_{u} \mathbf{Z}^{\prime} \mathbf{A}^{12} & \mathbf{Z}^{\prime} \mathbf{W}_{2}^{\prime} \mathbf{W}_{2} \mathbf{Z}+\alpha_{u} \mathbf{Z}^{\prime} \mathbf{A}^{22} \mathbf{Z}+\mathbf{D}^{-1} \sigma_{e}^{2} & \alpha_{u} \mathbf{Z}^{\prime} \\
\mathbf{0} & \mathbf{0} & \alpha_{u} \mathbf{Z} & \alpha_{u} \mathbf{A}_{22}
\end{array}\right]\left[\begin{array}{c}
\hat{\mathbf{b}} \\
\hat{\mathbf{u}}_{1} \\
\hat{\mathbf{g}} \\
-\hat{\mathbf{\varphi}}
\end{array}\right]=\left[\begin{array}{c}
\mathbf{X}^{\prime} \mathbf{y} \\
\mathbf{W}_{1}^{\prime} \mathbf{y}_{1} \\
\mathbf{Z}^{\prime} \mathbf{W}_{2}^{\prime} \mathbf{y}_{2} \\
\mathbf{0}
\end{array}\right]
$$

These equations are nevertheless more cumbersome than [4], [5], or [7].

\section{APPENDIX B}

Here, we provide details about the equivalent derivation of the MME. We start from the model

$$
\mathbf{y}=\mathbf{X b}+\mathbf{W}\left(\mathbf{u}^{*}+\mathbf{d}\right)+\mathbf{e} .
$$

According to the assumptions in the main text, the corresponding MME are

$$
\left[\begin{array}{cccc}
\mathbf{X}^{\prime} \mathbf{X} & \mathbf{X}_{1}^{\prime} \mathbf{W}_{1} & \mathbf{X}_{2}^{\prime} \mathbf{W}_{2} & \mathbf{X}_{1}^{\prime} \mathbf{W}_{1} \mathbf{A}_{12} \mathbf{A}_{22}^{-1}+\mathbf{X}_{2}^{\prime} \mathbf{W}_{2} \\
\mathbf{W}_{1}^{\prime} \mathbf{X}_{1} & \mathbf{W}_{1}^{\prime} \mathbf{W}_{1}+\alpha_{u} \mathbf{A}^{11} & \alpha_{u} \mathbf{A}^{12} & \mathbf{W}_{1}^{\prime} \mathbf{W}_{1} \mathbf{A}_{12} \mathbf{A}_{22}^{-1} \\
\mathbf{W}_{2}^{\prime} \mathbf{X}_{2} & \alpha_{u} \mathbf{A}^{21} & \mathbf{W}_{2}^{\prime} \mathbf{W}_{2}+\alpha_{u} \mathbf{A}^{22} & \mathbf{W}_{2}^{\prime} \mathbf{W}_{2} \\
\left\{\mathbf{A}_{22}^{-1} \mathbf{A}_{21} \mathbf{W}_{1}^{\prime} \mathbf{X}_{1}\right. & \mathbf{A}_{22}^{-1} \mathbf{A}_{21} \mathbf{W}_{1}^{\prime} \mathbf{W}_{1} & \mathbf{W}_{2}^{\prime} \mathbf{W}_{2} & \left\{\mathbf{A}_{22}^{-1} \mathbf{A}_{21} \mathbf{W}_{1} \mathbf{W}_{1}^{\prime} \mathbf{A}_{12} \mathbf{A}_{22}^{-1}\right. \\
\left.+\mathbf{W}_{2}^{\prime} \mathbf{X}_{2}\right\} & & & \left.\mathbf{W}_{2}^{\prime} \mathbf{W}_{2}+\alpha_{u}\left(\mathbf{G}-\mathbf{A}_{22}\right)^{-\mathbf{1}}\right\}
\end{array}\right]\left[\begin{array}{c}
\hat{\mathbf{b}} \\
\hat{\mathbf{u}}_{1}^{*} \\
\hat{\mathbf{u}}_{2}^{*} \\
\hat{\mathbf{d}}_{2}
\end{array}\right]=\left[\begin{array}{c}
\mathbf{X y} \\
\mathbf{W}_{1}^{\prime} \mathbf{y}_{1} \\
\mathbf{W}_{2}^{\prime} \mathbf{y}_{2} \\
\mathbf{A}_{22}^{-1} \mathbf{A}_{21} \mathbf{W}_{1}^{\prime} \mathbf{y}_{1} \\
\left.+\mathbf{W}_{2}^{\prime} \mathbf{y}_{2}\right\}
\end{array}\right] .
$$

Now, mimicking the development of Quaas and Pollak (1981) for groups of unknown parents, let

$$
\mathbf{S}=\left[\begin{array}{cccc}
\mathbf{I} & \mathbf{0} & \mathbf{0} & \mathbf{0} \\
\mathbf{0} & \mathbf{I} & \mathbf{0} & \mathbf{A}_{12} \mathbf{A}_{22}^{-1} \\
\mathbf{0} & \mathbf{0} & \mathbf{I} & \mathbf{I} \\
\mathbf{0} & \mathbf{0} & \mathbf{0} & \mathbf{I}
\end{array}\right]
$$

and

$$
\mathbf{S}^{-1}=\left[\begin{array}{cccc}
\mathbf{I} & \mathbf{0} & \mathbf{0} & \mathbf{0} \\
\mathbf{0} & \mathbf{I} & \mathbf{0} & -\mathbf{A}_{12} \mathbf{A}_{22}^{-1} \\
\mathbf{0} & \mathbf{0} & \mathbf{I} & -\mathbf{I} \\
\mathbf{0} & \mathbf{0} & \mathbf{0} & \mathbf{I}
\end{array}\right]
$$

Inserting $\mathbf{S}^{-1} \mathbf{S}$ between the coefficient matrix and the vector of unknowns and premultiplying both sides of the system by $\mathbf{S}^{-\mathbf{T}}$, we get 
and

$$
\left[\begin{array}{c}
\hat{\mathbf{b}} \\
\hat{\mathbf{u}}_{1} \\
\hat{\mathbf{u}}_{2} \\
\hat{\mathbf{d}}_{2}
\end{array}\right]=\left[\begin{array}{cccc}
\mathbf{I} & \mathbf{0} & \mathbf{0} & \mathbf{0} \\
\mathbf{0} & \mathbf{I} & \mathbf{0} & \mathbf{A}_{12} \mathbf{A}_{22}^{-\mathbf{1}} \\
\mathbf{0} & \mathbf{0} & \mathbf{I} & \mathbf{I} \\
\mathbf{0} & \mathbf{0} & \mathbf{0} & \mathbf{I}
\end{array}\right]\left[\begin{array}{c}
\hat{\mathbf{b}} \\
\hat{\mathbf{u}}_{1}^{*} \\
\hat{\mathbf{u}}_{2}^{*} \\
\hat{\mathbf{d}}_{2}
\end{array}\right]
$$

$$
\left[\begin{array}{cccc}
\mathbf{X}^{\prime} \mathbf{X} & \mathbf{W}_{1}^{\prime} \mathbf{W}_{1} & \mathbf{X}_{2}^{\prime} \mathbf{W}_{2} & \mathbf{0} \\
\mathbf{W}_{1}^{\prime} \mathbf{X}_{1} & \mathbf{W}_{1}^{\prime} \mathbf{W}_{1}+\alpha_{u} \mathbf{A}^{11} & \alpha_{u} \mathbf{A}^{12} & -\alpha_{u}\left(\mathbf{A}^{11} \mathbf{A}_{12} \mathbf{A}_{22}^{-1}+\mathbf{A}^{12}\right) \\
\mathbf{W}_{2}^{\prime} \mathbf{X}_{2} & \alpha_{u} \mathbf{A}^{21} & \mathbf{W}_{2}^{\prime} \mathbf{W}_{2}+\alpha_{u} \mathbf{A}^{22} & -\alpha_{u}\left(\mathbf{A}^{11} \mathbf{A}_{12} \mathbf{A}_{22}^{-1}+\mathbf{A}^{22}\right) \\
& & & \alpha_{u}\left\{\left(\mathbf{A}_{\mathbf{2 2}}^{-1} \mathbf{A}_{21} \mathbf{A}^{11} \mathbf{A}_{12} \mathbf{A}_{22}^{-1}+\mathbf{A}^{12}\right)\right. \\
\mathbf{0} & -\alpha_{u}\left(\mathbf{A}_{\mathbf{2 2}}^{-1} \mathbf{A}_{21} \mathbf{A}^{11}+\mathbf{A}^{21}\right) & -\alpha_{u}\left(\mathbf{A}_{\mathbf{2}}^{-1} \mathbf{A}_{21} \mathbf{A}^{11}+\mathbf{A}^{22}\right) & +\left(\mathbf{A}^{11} \mathbf{A}_{12} \mathbf{A}_{\mathbf{2}}^{-1}+\mathbf{A}^{22}\right) \\
& & \left.+\left(\mathbf{G}-\mathbf{A}_{22}\right)^{-\mathbf{1}}\right\}
\end{array}\right]\left[\begin{array}{c}
\hat{\mathbf{b}} \\
\hat{\mathbf{u}}_{1} \\
\hat{\mathbf{u}}_{2} \\
\hat{\mathbf{d}}_{2}
\end{array}\right]=\left[\begin{array}{c}
\mathbf{X}^{\prime} \mathbf{y} \\
\mathbf{W}_{1}^{\prime} \mathbf{y}_{1} \\
\mathbf{W}_{2}^{\prime} \mathbf{y}_{2} \\
\mathbf{0}
\end{array}\right]
$$

The rules for the inverse of a partition matrix (Searle, 1982) imply that $\mathbf{A}^{11} \mathbf{A}_{12} \mathbf{A}_{22}^{-1}+\mathbf{A}^{12}=\mathbf{0}$ and $\mathbf{A}^{22}+\mathbf{A}_{22}^{-1} \mathbf{A}_{21} \mathbf{A}^{11} \mathbf{A}_{12} \mathbf{A}_{22}^{-1}=\mathbf{A}_{22}^{-1}$. Therefore, the system above simplifies to

$$
\left[\begin{array}{cccc}
\mathbf{X}^{\prime} \mathbf{X} & \mathbf{W}_{1}^{\prime} \mathbf{W}_{1} & \mathbf{X}_{2}^{\prime} \mathbf{W}_{2} & \mathbf{0} \\
\mathbf{W}_{1}^{\prime} \mathbf{X}_{1} & \mathbf{W}_{1}^{\prime} \mathbf{W}_{1}+\alpha_{u} \mathbf{A}^{11} & \alpha_{u} \mathbf{A}^{12} & \mathbf{0} \\
\mathbf{W}_{2}^{\prime} \mathbf{X}_{2} & \alpha_{u} \mathbf{A}^{21} & \mathbf{W}_{2}^{\prime} \mathbf{W}_{2}+\alpha_{u} \mathbf{A}^{22} & -\alpha_{u} \mathbf{A}_{22}^{-1} \\
\mathbf{0} & \mathbf{0} & -\alpha_{u} \mathbf{A}_{22}^{-1} & \alpha_{u}\left\{\mathbf{A}_{22}^{-1}+\left(\mathbf{G}-\mathbf{A}_{22}\right)^{-1}\right\}
\end{array}\right]\left[\begin{array}{c}
\hat{\mathbf{b}} \\
\hat{\mathbf{u}}_{1} \\
\hat{\mathbf{u}}_{2} \\
\hat{\mathbf{d}}_{2}
\end{array}\right]=\left[\begin{array}{c}
\mathbf{X}^{\prime} \mathbf{y} \\
\mathbf{W}_{1}^{\prime} \mathbf{y}_{1} \\
\mathbf{W}_{2}^{\prime} \mathbf{y}_{2} \\
\mathbf{0}
\end{array}\right] .
$$

Absorbing the last equation of system, we get

$$
\left[\begin{array}{ccc}
\mathbf{X}^{\prime} \mathbf{X} & \mathbf{X}_{1}^{\prime} \mathbf{W}_{1} & \mathbf{X}_{2}^{\prime} \mathbf{W}_{2} \\
\mathbf{W}_{1}^{\prime} \mathbf{X}_{1} & \mathbf{W}_{1}^{\prime} \mathbf{W}_{1}+\alpha_{u} \mathbf{A}^{11} & \alpha_{u} \mathbf{A}^{12} \\
\mathbf{W}_{2}^{\prime} \mathbf{X}_{2} & \alpha_{u} \mathbf{A}^{21} & \mathbf{W}_{2}^{\prime} \mathbf{W}_{2}+\alpha_{u}\left(\mathbf{A}^{22}-\mathbf{C}\right)
\end{array}\right]\left[\begin{array}{c}
\hat{\mathbf{b}} \\
\hat{\mathbf{u}}_{1} \\
\hat{\mathbf{u}}_{2}
\end{array}\right]=\left[\begin{array}{c}
\mathbf{X}_{1}^{\prime} \mathbf{y}_{1}+\mathbf{X}_{2}^{\prime} \mathbf{y}_{2} \\
\mathbf{W}_{1}^{\prime} \mathbf{y}_{1} \\
\mathbf{W}_{2}^{\prime} \mathbf{y}_{2}
\end{array}\right],
$$

with $\mathbf{C}=\mathbf{A}_{22}^{-1}\left[\mathbf{A}_{22}^{-1}+\left(\mathbf{G}-\mathbf{A}_{22}\right)^{-1}\right]^{-1} \mathbf{A}_{22}^{-1}=\left[\mathbf{A}_{22}+\mathbf{A}_{22}\left(\mathbf{G}-\mathbf{A}_{22}\right)^{-1} \mathbf{A}_{22}\right]^{-1}$.

Let a matrix

$$
\mathbf{K}=\left[\begin{array}{ll}
\mathbf{K}_{11} & \mathbf{K}_{12} \\
\mathbf{K}_{21} & \mathbf{K}_{22}
\end{array}\right]=\left[\begin{array}{cc}
\mathbf{A}_{22} & \mathbf{A}_{22} \\
\mathbf{A}_{22} & -\left(\mathbf{G}-\mathbf{A}_{22}\right)
\end{array}\right]
$$

and

$$
\mathbf{K}^{-1}=\left[\begin{array}{ll}
\mathbf{K}^{11} & \mathbf{K}^{12} \\
\mathbf{K}^{21} & \mathbf{K}^{22}
\end{array}\right]
$$

Applying the rules for the inverse of a partition matrix (Searle, 1982) to matrix K leads to

$$
\mathbf{K}^{22}=\left[-\left(\mathbf{G}-\mathbf{A}_{22}\right)-\mathbf{A}_{22} \mathbf{A}_{22}^{-1} \mathbf{A}_{22}\right]^{-1}=\left[-\mathbf{G}+\mathbf{A}_{22}-\mathbf{A}_{22}\right]^{-1}=-\mathbf{G}^{-1}
$$


and

$$
\begin{aligned}
\mathbf{K}^{11} & =\left[\mathbf{A}_{22}-\mathbf{A}_{22}\left\{-\left(\mathbf{G}-\mathbf{A}_{22}\right)^{-1}\right\} \mathbf{A}_{22}\right]^{-1}=\mathbf{C} \\
& =\mathbf{A}_{22}^{-1}+\mathbf{A}_{22}^{-1} \mathbf{A}_{22} \mathbf{G}^{-1} \mathbf{A}_{22} \mathbf{A}_{22}^{-1}=-\left(\mathbf{G}^{-1}-\mathbf{A}_{22}^{-1}\right) .
\end{aligned}
$$

Replacing $\mathbf{C}$ in [B3] by expression [B4] gives the MME [3].

\section{APPENDIX C}

In this appendix, we show how the problem of lack of positive definiteness of $\mathbf{G}-\mathbf{A}$ can be circumvented. Consider the model

$$
\mathbf{y}=\mathbf{X b}+\mathbf{Z u}+\mathbf{e}
$$

with $\operatorname{Var}(\mathbf{u})=\mathbf{G} \sigma_{u}^{2}$.

We want to consider an equivalent model where $\mathbf{u}$ is decomposed into uncorrelated $\mathbf{u}^{*}+\mathbf{d}$ with $\operatorname{Var}\left(\mathbf{u}^{*}\right)=\mathbf{A} \sigma_{u}^{2}$. and $\operatorname{Var}(\mathbf{d})=(\mathbf{G}-\mathbf{A}) \sigma_{u}^{2}$, where $\mathbf{A}$ is the numerator relationship matrix.

Let $\boldsymbol{P}=\mathbf{G}-\mathbf{A}=\mathbf{V} \mathbf{D} \mathbf{V}^{\prime}$ with

$$
\mathbf{D}=\left[\begin{array}{cc}
\mathbf{D}_{+} & \mathbf{0} \\
\mathbf{0} & -\mathbf{D}_{-}
\end{array}\right] \text {. }
$$

Matrices $\mathbf{D}_{+}$and $\mathbf{D}_{-}$are positive diagonal matrices of size $n+$ and $n-$. Decompose the matrix of eigenvectors accordingly: $\mathbf{V}=\left[\mathrm{V}_{+} \mathrm{V}_{-}\right]$. By definition, $\mathbf{V V}^{\prime}=\mathbf{V}^{\prime} \mathbf{V}=\mathbf{I}$ or

$$
\left[\mathbf{V}_{+} \mathbf{V}_{-}\right]\left[\begin{array}{c}
\mathbf{V}_{+}^{\prime} \\
\mathbf{V}_{-}^{\prime}
\end{array}\right]=\mathbf{V}_{+} \mathbf{V}_{+}^{\prime}+\mathbf{V}_{-} \mathbf{V}_{-}^{\prime}=\mathbf{I}
$$

and

$$
\left[\begin{array}{c}
\mathbf{V}_{+}^{\prime} \\
\mathbf{V}_{-}^{\prime}
\end{array}\right]\left[\mathbf{V}_{+} \mathbf{V}_{-}\right]=\left[\begin{array}{cc}
\mathbf{V}_{+}^{\prime} \mathbf{V}_{+} & \mathbf{0} \\
\mathbf{0} & \mathbf{V}_{-}^{\prime} \mathbf{V}_{-}
\end{array}\right]=\left[\begin{array}{cc}
\mathbf{I}_{n+} & \mathbf{0} \\
\mathbf{0} & \mathbf{I}_{n-}
\end{array}\right]
$$

We have

$$
\mathbf{u}=\mathbf{u}^{*}+\mathbf{d}
$$

Rewrite

$$
\mathbf{d}=\left[\begin{array}{ll}
\mathbf{V}_{+} & -i \mathbf{V}_{-}
\end{array}\right]\left[\begin{array}{l}
\mathbf{V}_{+}^{\prime} \\
i \mathbf{V}_{-}^{\prime}
\end{array}\right] \mathbf{d}=\left[\begin{array}{ll}
\mathbf{V}_{+} & -i \mathbf{V}_{-}
\end{array}\right] \boldsymbol{\delta}
$$

with

$$
\boldsymbol{\delta}=\left[\begin{array}{l}
\boldsymbol{\delta}_{+} \\
\boldsymbol{\delta}_{-}
\end{array}\right]
$$

(note: $\mathbf{d}$ and $\boldsymbol{\delta}_{+}$are vectors of real numbers and $\boldsymbol{\delta}_{-}$is a vector of imaginary numbers). Assume 


$$
\delta \sim \mathbf{N}\left(\mathbf{0},\left[\begin{array}{cc}
\mathbf{D}_{+} & \mathbf{0} \\
\mathbf{0} & \mathbf{D}_{-}
\end{array}\right] \sigma_{u}^{2}\right)
$$

and $\operatorname{cov}\left(\mathbf{u}, \boldsymbol{\delta}^{\prime}\right)=\mathbf{0}$.

Rewrite $[\mathrm{C} 2]$ as

$$
\mathbf{u}=\mathbf{u}^{*}+\mathbf{d}=\mathbf{u}^{*}+\mathbf{V}_{+} \delta_{+}-i \mathbf{V}_{-} \boldsymbol{\delta}_{-} .
$$

We have

$$
E[\mathbf{y}]=\mathbf{X b}+\mathbf{Z} E\left[\mathbf{u}^{*}\right]+\mathbf{Z V}_{+} E\left[\boldsymbol{\delta}_{+}\right]-\mathbf{i} \mathbf{Z} \mathbf{V}_{-} E\left[\boldsymbol{\delta}_{-}\right]+E[\mathbf{e}]=\mathbf{X b}
$$

and

$$
\begin{aligned}
& \operatorname{Var}[\mathbf{y}]=\mathbf{Z}\left[\operatorname{Var}\left(\mathbf{u}^{*}\right)+\mathbf{V}_{+} \operatorname{Var}\left(\boldsymbol{\delta}_{+}\right) \mathbf{V}_{+}^{\prime}+i^{2} \mathbf{V}_{-} \operatorname{Var}\left(\boldsymbol{\delta}_{-}\right) \mathbf{V}_{-}^{\prime}\right] \mathbf{Z}^{\prime}+\operatorname{Var}[\mathbf{e}] \\
& =\mathbf{Z}\left[\mathbf{A}+\mathbf{V}_{+} \mathbf{D}_{+} \mathbf{V}_{+}^{\prime}+i^{2} \mathbf{V}_{-} \mathbf{D}_{-} \mathbf{V}_{-}^{\prime}\right] \mathbf{Z}^{\prime} \sigma_{u}^{2}+\mathbf{I} \sigma_{e}^{2} \\
& =\mathbf{Z}\left[\mathbf{A}+\left[\begin{array}{ll}
\mathbf{V}_{+} & \mathbf{V}_{-}
\end{array}\right]\left[\begin{array}{cr}
\mathbf{D}_{+} & \mathbf{0} \\
\mathbf{0} & -\mathbf{D}_{-}
\end{array}\right]\left[\begin{array}{c}
\mathbf{V}_{+}^{\prime} \\
\mathbf{V}_{-}^{\prime}
\end{array}\right]\right] \mathbf{Z}^{\prime} \sigma_{u}^{2}+\mathbf{I} \sigma_{e}^{2} \\
& =\mathbf{Z}\left[\mathbf{A}+\mathbf{V D} \mathbf{V}^{\prime}\right] \mathbf{Z}^{\prime} \sigma_{u}^{2}+\mathbf{I} \sigma_{e}^{2} \\
& =\mathbf{Z}[\mathbf{A}+(\mathbf{G}-\mathbf{A})] \mathbf{Z}^{\prime} \sigma_{u}^{2}+\mathbf{I} \sigma_{e}^{2} \\
& =\mathbf{Z G} \mathbf{Z}^{\prime} \sigma_{u}^{2}+\mathbf{I} \sigma_{e}^{2} .
\end{aligned}
$$

Therefore, the model with $\mathbf{u}^{*}$ and $\boldsymbol{\delta}$ is equivalent to the model with $\mathbf{u}^{*}$ and $\mathbf{d}$.

The model with $\mathbf{u}^{*}$ and $\boldsymbol{\delta}$ leads to usual MME, where the variance of $\boldsymbol{\delta}$ is the positive definite matrix

$$
\left[\begin{array}{cc}
\mathbf{D}_{+} & \mathbf{0} \\
\mathbf{0} & \mathbf{D}_{-}
\end{array}\right] \sigma_{u}^{2} .
$$

Regular MME are usually derived for variables with real domain. However, in this case, we deal with $\boldsymbol{\delta}_{-}$being imaginary random effects. Because $\boldsymbol{\delta}_{+}$and $\boldsymbol{\delta}_{-}$are uncorrelated and, further, they are either real or imaginary (but not complex numbers), they vary according to the so-called circular symmetric complex normal distribution, for which, roughly speaking, regular expressions of multivariate normality for real numbers apply (Picinbono, 1996). Indeed, some derivations of BLUP (Henderson, 1973) do not involve normality or even probability distribution functions, but only covariance matrices. These MME are

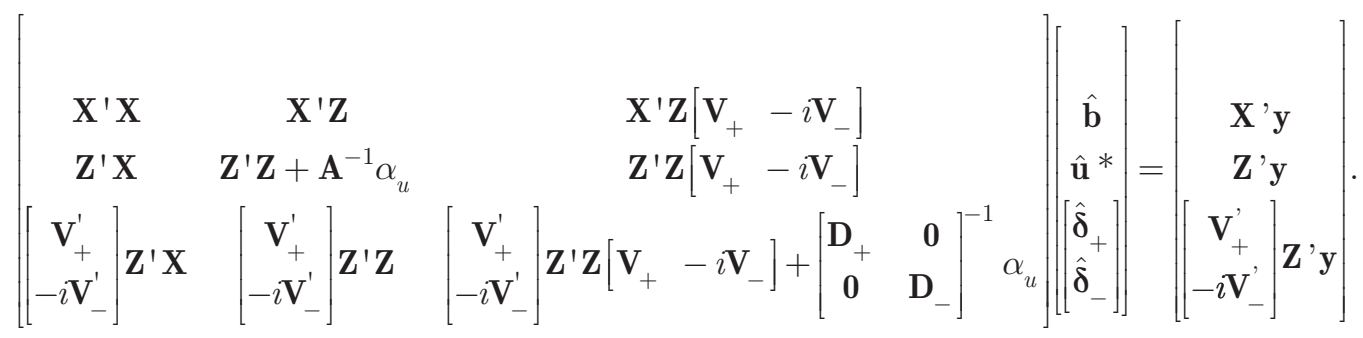

Using

$$
\begin{gathered}
\mathbf{d}=\left[\begin{array}{ll}
\mathbf{V}_{+} & -\mathbf{i} \mathbf{V}_{-}
\end{array}\right] \mathbf{\delta} \\
{\left[\begin{array}{ll}
\mathbf{V}_{+} & i \mathbf{V}_{-}
\end{array}\right]\left[\begin{array}{c}
\mathbf{V}_{+}^{\prime} \\
-i \mathbf{V}_{-}^{\prime}
\end{array}\right]=\mathbf{I},}
\end{gathered}
$$


and

$$
\left[\begin{array}{c}
\mathbf{V}_{+}^{\prime} \\
\imath \mathbf{V}_{-}^{\prime}
\end{array}\right]\left[\begin{array}{ll}
\mathbf{V}_{+} & -\imath \mathbf{V}_{-}
\end{array}\right]=\mathbf{I},
$$

premultiply both sides of these MME by

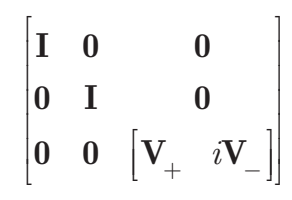

and insert

$$
\left.\mathbf{I}=\left[\begin{array}{ccc}
\mathbf{I} & \mathbf{0} & \mathbf{0} \\
\mathbf{0} & \mathbf{I} & \mathbf{0} \\
\mathbf{0} & \mathbf{0} & {\left[\begin{array}{c}
\mathbf{V}_{+}^{\prime} \\
\imath \mathbf{V}_{-}^{\prime}
\end{array}\right]}
\end{array}\right]\left[\begin{array}{llll}
\mathbf{I} & \mathbf{0} & \mathbf{0} \\
\mathbf{0} & \mathbf{I} & \mathbf{0} \\
\mathbf{0} & \mathbf{0} & {\left[\mathbf{V}_{+}\right.} & -\imath \mathbf{V}_{-}
\end{array}\right]\right]
$$

between the coefficient matrix and the solution vector. We get

$$
\left[\begin{array}{ccc}
\mathbf{X}^{\prime} \mathbf{X} & \mathbf{X}^{\prime} \mathbf{Z} & \mathbf{X}^{\prime} \mathbf{Z} \\
\mathbf{Z}^{\prime} \mathbf{X} & \mathbf{Z}^{\prime} \mathbf{Z}+\mathbf{A}^{-1} \alpha & \mathbf{Z}^{\prime} \mathbf{Z} \\
\mathbf{Z}^{\prime} \mathbf{X} & \mathbf{Z}^{\prime} \mathbf{Z} & \mathbf{Z}^{\prime} \mathbf{Z}+\alpha_{u}(\mathbf{G}-\mathbf{A})^{-1}
\end{array}\right]\left[\begin{array}{c}
\hat{\mathbf{b}} \\
\hat{\mathbf{u}} * \\
\hat{\mathbf{d}}
\end{array}\right]=\left[\begin{array}{c}
\mathbf{X}^{\prime} \mathbf{y} \\
\mathbf{Z}^{\prime} \mathbf{y} \\
\mathbf{Z}^{\prime} \mathbf{y}
\end{array}\right] .
$$

Now, this shows that if we have a model [C2] such that $\operatorname{var}(\mathbf{d})$ is not positive definite, there is an equivalent model [C3] in which all variance matrices are positive definite, which leads to the same MME [C4]. The only requisite is that the covariance matrix be nonsingular (i.e., invertible). In other words, there is no need to care whether $\mathbf{G}-\mathbf{A}$ is positive definite or not. 